\title{
Derived Picard groups of selfinjective Nakayama algebras.
}

\author{
Yury Volkov and Alexandra Zvonareva
}

To the memory of Alexander Ivanov

\begin{abstract}
In our proceeding paper a generating set of the derived Picard group of a selfinjective Nakayama algebra was constructed combining some previous results for Brauer tree algebras and the technique of orbit categories developed there. In this paper we finish the computation of the derived Picard group of a selfinjective Nakayama algebra.
\end{abstract}

\section{Introduction}

The derived Picard group $\operatorname{Tr} \operatorname{Pic}(A)$ of an algebra $A$ is the group of standard autoequivalences of the derived category modulo natural isomorphisms. This group is an interesting invariant of the derived equivalence connected to various other invariants such as Hochschild cohomology [1], the identity component of the algebraic group of outer automorphisms [2] and the group of autoequivalences of the stable category in the case of a selfinjective algebra.

The derived Picard group was computed in a few cases. If $A$ is local or commutative with a connected spectrum, then $\operatorname{Tr} \operatorname{Pic}(A) \simeq \operatorname{Pic}(A) \times \mathbb{Z}$ (see [3], [4]). In the work of Miyachi and Yekutieli the derived Picard group was computed for finite dimensional hereditary algebras [5]. In the work of Lenzing and Meltzer [6] the group of autoequivalences of the bounded derived category of a canonical algebra was studied and in the work of Broomhead, Pauksztello and Ploog this group was described for discrete derived categories [7].

The derived Picard group of a symmetric Nakayama algebra or an algebra derived equivalent to a symmetric Nakayama algebra was studied in many papers. Rouquier and Zimmermann defined a braid group action on the derived category of a Brauer line algebra with multiplicity 1 (see [3]). Later, Zimmermann investigated the case of arbitrary multiplicity [8]. Schaps and Zakay-Illouz defined an action of a braid group corresponding to $\widetilde{A}_{n-1}$ on the derived category of a Brauer star algebra with arbitrary multiplicity [9]. Note that the class of Brauer star algebras with arbitrary multiplicity coincides with the class of symmetric Nakayama algebras. Khovanov and Seidel developed the theory of spherical twists in [10], it follows from [10] and [11] that the braid group action defined in [3] is faithful. In [12] Muchtadi-Alamsyah proved that the braid group action defined in [9] is faithful in the case of multiplicity 1. In [13] the second named author computed the generating set of $\operatorname{Tr} \operatorname{Pic}(A)$ for a Brauer star algebra with arbitrary multiplicity. In [14] both authors extended this result to the class of selfinjective Nakayama algebras. 
Let $\mathbf{k}$ be an algebraically closed field and $A$ be a selfinjective Nakayama algebra over $\mathbf{k}$. The aim of this work is to describe the derived Picard group of $A$. The paper is organized as follows: in Sections 2.1 and 2.2 we collect some preliminary results on standard derived equivalences and the derived Picard group. In Section 2.3 we recall the technique of orbit categories or smash products developed in [14], which is used in Section 3.7.

In Section 3 we formulate and prove our main result: the description of the derived Picard group for selfinjective Nakayama algebras.

Let $m, n, t>0$ be some integers. We suppose that $n$ and $t$ are coprime. Let $\mathcal{Q}_{n m}$ be a cyclic quiver with $n m$ vertices. Let $\mathcal{I}_{n m, t m}$ be an ideal in the path algebra of $\mathcal{Q}_{n m}$ generated by all paths of length $t m+1$. We denote $\mathcal{N}_{n m, t m}:=\mathbf{k} \mathcal{Q}_{n m} / \mathcal{I}_{n m, t m}$.

Theorem. 1) If $m=1$, then

$$
\operatorname{Tr} \operatorname{Pic}\left(\mathcal{N}_{n m, t m}\right)=\operatorname{Tr} \operatorname{Pic}\left(\mathcal{N}_{n, t}\right) \cong C_{n} \times \mathcal{S}_{\left\lfloor\frac{t+n-1}{n}\right\rfloor}(\mathbf{k}) \times C_{\infty}
$$

2) If $m>1$ and $t=1$, then

$$
\operatorname{Tr} \operatorname{Pic}\left(\mathcal{N}_{n m, t m}\right)=\operatorname{Tr} \operatorname{Pic}\left(\mathcal{N}_{n m, m}\right) \cong \mathcal{A}_{m, n} \times \mathbf{k}^{*}
$$

3) If $m>1$ and $t>1$, then

$$
\operatorname{Tr} \operatorname{Pic}\left(\mathcal{N}_{n m, t m}\right) \cong\left(\mathcal{B}\left(\widetilde{A}_{m-1}\right) \rtimes_{\varphi_{m, n}} C_{n m}\right) \times \mathcal{S}_{\left\lfloor\frac{t+n-1}{n}\right\rfloor}(\mathbf{k}) \times C_{\infty}
$$

The corresponding groups are defined in Section 2.4. In Section 3.1 we describe the Picard group of a selfinjective Nakayama algebra, in Section 3.2 we describe the generating set of $\operatorname{Tr} \operatorname{Pic}(A)$ and prove the first part of the theorem. In Sections 3.3 and 3.4 we describe an algebra $\mathcal{R}$, which is derived equivalent to $\mathcal{N}_{n m, m}$. This algebra was studied in [15], there a faithful action of a braid group was defined, providing a generalisation of the results from [10], using this result we prove the second part of the theorem in Section 3.5. In Section 3.6 we recall some facts about the affine braid group $\mathcal{B}\left(\widetilde{A}_{n-1}\right)$ and it's faithful action on the derived category in some cases. Also one nice monomorphism of affine braid groups is described in this section. In Section 3.7 we extend the faithfulness to the general case, this allows us to prove the third part of the theorem in Section 3.8 .

Acknowledgement: We would like to thank Semën Podkorytov and Mikhail Basok for discussions on braid groups. The work of the first named author was partially supported by the Russian Foundation for Basic Research (RFFI grant 13-01-00902) and by the SãoPaulo Research Foundation (proc. 2014/19521-3), the work of the second named author was partially supported by the Russian Foundation for Basic Research (RFFI grant 13-0100902) and by the Chebyshev Laboratory (Department of Mathematics and Mechanics, Saint Petersburg State University) under Russian Federation Government grant 11.G34.31.0026.

\section{Preliminaries}

\subsection{Standard derived equivalences and the derived Picard group}

Throughout this paper $\mathbf{k}$ is an algebraically closed field and we simply write $\otimes$ instead of $\otimes_{\mathbf{k}}$. Let $A$ and $B$ be $\mathbf{k}$-algebras. All modules are right modules if otherwise is not stated. 
We denote by $\mathcal{C} A$ the category of $A$-complexes. By $\mathcal{K} A$ we denote the homotopy category of $\mathcal{C} A$. By $\mathcal{K}_{p}^{\mathrm{b}} A$ we denote the subcategory of $\mathcal{K} A$ of bounded complexes of finitely generated projective modules. By $\mathcal{D} A$ we denote the derived category of $\mathcal{K} A$. The algebras $A$ and $B$ are called derived equivalent if their derived categories are equivalent as triangulated categories. It is well known that if $A$ and $B$ are derived equivalent then there are a complex of $B^{\mathrm{op}} \otimes A$ modules $X$ and a complex of $A^{\text {op }} \otimes B$-modules $Y$ such that $X \otimes_{A} Y \cong B$ in $\mathcal{D}\left(B^{\text {op }} \otimes B\right)$ and $Y \otimes_{B} X \cong A$ in $\mathcal{D}\left(A^{\text {op }} \otimes A\right)$ [16]. In this case $X$ is called a two-sided tilting complex. The complex $X$ defines an equivalence $T_{X}=-\otimes_{B} X: \mathcal{D} B \rightarrow \mathcal{D} A$ (which is quasi-inverse to $T_{Y}$ ). It is well-known that the equivalence $T_{X}$ induces an equivalence from $\mathcal{K}_{p}^{\mathrm{b}} B$ to $\mathcal{K}_{p}^{\mathrm{b}} A$. The equivalence $T_{X}: \mathcal{K}_{p}^{\mathrm{b}} B \rightarrow \mathcal{K}_{p}^{\mathrm{b}} A$ is defined modulo natural isomorphism by the isomorphism class of $X$ in $\mathcal{D}\left(A^{\text {op }} \otimes B\right)$. Moreover, if $T_{X} \cong T_{X^{\prime}}$, then $X \cong X^{\prime}$ in $\mathcal{D}\left(B^{\text {op }} \otimes A\right)$.

Definition 1. The equivalences of the form $T_{X}$ are called standard equivalences. The set of isomorphism classes of two-sided tilting $A^{\text {op }} \otimes A$-complexes forms a group with respect to the operation $\otimes_{A}$. This group is called the derived Picard group of $A$ and we denote it by $\operatorname{TrPic}(A)$. As it was mentioned above the group $\operatorname{TrPic}(A)$ can be considered as a group of standard autoequivalences of $\mathcal{K}_{p}^{\mathrm{b}} A$ modulo natural isomorphisms.

\subsection{Standard derived equivalences and tilting functors}

It is not very convenient to work with complexes of bimodules. To circumvent this problem we need the following definitions.

Definition 2. A complex $H \in \mathcal{K}_{p}^{\mathrm{b}} A$ is called a tilting complex for $A$ if the following two conditions are satisfied

1. $\mathcal{K}_{p}^{\mathrm{b}} A(H, H[i])=0$ for all $i \neq 0$;

2. the minimal triangulated subcategory of $\mathcal{K}_{p}^{\mathrm{b}} A$ which contains $H$ and is closed under isomorphisms, direct summands and direct sums contains $A$.

A tilting functor from $B$ to $\mathcal{K}_{p}^{\mathrm{b}} A$ is a pair $(H, \theta)$ where $H$ is a tilting complex for $A$ and $\theta: B \rightarrow \operatorname{End}_{\mathcal{K}_{p}^{\mathrm{b}} A}(H, H)$ is an isomorphism of algebras. We say that $(H, \theta)$ is isomorphic to $\left(H^{\prime}, \theta^{\prime}\right)$ if there is an isomorphism $\xi \in \mathcal{K}_{p}^{\mathrm{b}} A\left(H, H^{\prime}\right)$ such that $\xi \theta(b)=\theta^{\prime}(b) \xi$ for all $b \in B$. Sometimes we simply write $\theta$ instead of $(H, \theta)$ if $H$ is clear.

Every standard equivalence $T_{X}: \mathcal{K}_{p}^{\mathrm{b}} B \rightarrow \mathcal{K}_{p}^{\mathrm{b}} A$ defines a tilting functor $\left(H_{X}, \theta_{X}\right)$, since $B$ is an object of $\mathcal{K}_{p}^{\mathrm{b}} B$ and $B \cong \operatorname{End}_{\mathcal{K}_{p}^{\mathrm{b}} B}(B, B) \cong \operatorname{End}_{\mathcal{K}_{p}^{\mathrm{b}} A}\left(T_{X}(B), T_{X}(B)\right)$. Conversely, if $(H, \theta)$ is a tilting functor, then there is a two-sided tilting complex $X$ such that $\left(H_{X}, \theta_{X}\right) \cong(H, \theta)$ (see [17, Section 9]), but the construction of $X$ is tedious. We want to construct a functor $F_{\theta}: \mathcal{K}_{p}^{\mathrm{b}} B \rightarrow \mathcal{K}_{p}^{\mathrm{b}} A$ in such a way that $F_{\theta} \cong T_{X}$. This construction was introduced in [18] and is described in [14, Section 3] with a slight simplification. We recall some details of this construction here for convenience. Denote by $\mathcal{P}_{B}$ the category of finitely generated projective $B$-modules and by add $H$ the full subcategory of $\mathcal{K}_{p}^{\mathrm{b}} A$ formed by direct summands of finite direct sums of copies of $H$. Then $\theta$ induces an equivalence $S_{\theta}: \mathcal{P}_{B} \rightarrow \operatorname{add} H$. The construction of $S_{\theta}$ is described in [14. Note that the equivalence $S_{\theta}$ defines the tilting functor $\theta$. So sometimes we will define the tilting functor $(H, \theta)$ by the description of the equivalence $S_{\theta}$.

Suppose that we have constructed the functor $S_{\theta}$. Let us consider $U \in \mathcal{K}_{p}^{\mathrm{b}} B$. Then $U=\oplus_{i \in \mathbb{Z}} U_{i}$, where $U_{i} \in \mathcal{P}_{B}$. The object $V_{i}:=S\left(U_{i}\right) \in \operatorname{add} H(i \in \mathbb{Z})$ is defined and has 
some grading $V_{i}=\oplus_{j \in \mathbb{Z}} V_{i, j}$. The last formula defines a bigrading on $V:=S_{\theta}(U)=\oplus_{i \in \mathbb{Z}} V_{i}=$ $\oplus_{i, j \in \mathbb{Z}} V_{i, j}$. Note that $V_{i, j}=0$ for large enough and small enough $i$ or $j$. Let $d_{U}: U \rightarrow U$ be a differential of $U$. Then $d_{U}=\sum_{i \in \mathbb{Z}} d_{U, i}$, where $d_{U, i} \in \operatorname{Hom}_{B}\left(U_{i}, U_{i+1}\right)$. Let us choose some representative of $S_{\theta}\left(d_{U, i}\right)$ in $\mathcal{C} A\left(V_{i}, V_{i+1}\right)$ and denote it by $d_{1, i}$. Note that $d_{1, i}=\sum_{j \in \mathbb{Z}} d_{1, i, j}$ for some $d_{1, i, j}: V_{i, j} \rightarrow V_{i+1, j}$. We define $\tau_{1, i, j}:=(-1)^{i+j} d_{1, i, j}$ and $\tau_{1}=\sum_{i, j \in \mathbb{Z}} \tau_{1, i, j}$. Also for any $i \in \mathbb{Z}$ we have a differential $\tau_{0, i}=\sum_{j \in \mathbb{Z}} \tau_{0, i, j}: V_{i} \rightarrow V_{i}$, where $\tau_{0, i, j}=\left(d_{V_{i}}\right)_{j}: V_{i, j} \rightarrow V_{i, j+1}$. So we have constructed differentials $\tau_{0}=\sum_{i \in \mathbb{Z}} \tau_{0, i}, \tau_{1}: V \rightarrow V$ of degrees $(0,1)$ and $(1,0)$ respectively. These two differentials can be extended to a sequence $\left(\tau_{i}\right)_{i \geqslant 0}$ such that $\tau_{i}: V \rightarrow V$ is of degree $(i, 1-i)$ and $\sum_{i=0}^{l} \tau_{i} \tau_{l-i}=0$ for any $l \geqslant 0$. Let us now define $F_{\theta}(U) \in \mathcal{K}_{p}^{\mathrm{b}} A$. As an $A$-module $F_{\theta}(U)=V$. The $l$-th component of $F_{\theta}(U)$ is defined by the formula

$$
\left(F_{\theta}(U)\right)_{l}=\bigoplus_{i+j=l} V_{i, j}
$$

The differential of $F_{\theta}(U)$ is the map induced by the map $\tau=\sum_{i \geqslant 0} \tau_{i}: V \rightarrow V$. Consider $U, U^{\prime} \in \mathcal{K}_{p}^{\mathrm{b}} B$ and $f \in \mathcal{K}_{p}^{\mathrm{b}} B\left(U, U^{\prime}\right)$. Suppose that $f$ is represented by $\sum_{i \in \mathbb{Z}} f_{i}\left(f_{i}: U_{i} \rightarrow U_{i}^{\prime}\right)$ in $\mathcal{C} B\left(U, U^{\prime}\right)$. Let us introduce $V:=S_{\theta}(U), V^{\prime}:=S_{\theta}\left(U^{\prime}\right)$. The modules $V$ and $V^{\prime}$ are bigraded as before. Let $\left(\tau_{i}\right)_{i \geqslant 0}$ and $\left(\tau_{i}^{\prime}\right)_{i \geqslant 0}$ be the corresponding sequences from the construction of $F_{\theta}(U)$ and $F_{\theta}\left(U^{\prime}\right)$ respectively. For any $i \in \mathbb{Z}$ we choose some representative $\alpha_{0, i} \in \mathcal{C} A\left(V_{i}, V_{i}^{\prime}\right)$ of $S_{\theta}\left(f_{i}\right)$. Then we obtain a morphism $\alpha_{0}=\sum_{i \in \mathbb{Z}} \alpha_{0, i}: V \rightarrow V^{\prime}$ of degree $(0,0)$. It can be extended to a sequence $\left(\alpha_{i}\right)_{i \geqslant 0}$ such that $\alpha_{i}: V \rightarrow V^{\prime}$ is of degree $(i,-i)$ and $\sum_{i=0}^{l} \alpha_{i} \tau_{l-i}=\sum_{i=0}^{l} \tau_{i}^{\prime} \alpha_{l-i}$ for any $l \geqslant 0$. We define $F_{\theta}(f) \in \mathcal{K}_{p}^{\mathrm{b}} A\left(F_{\theta}(U), F_{\theta}\left(U^{\prime}\right)\right)$ as the class of the morphism $\alpha=\sum_{i \geqslant 0} \alpha_{i}: V \rightarrow V^{\prime}$. It can be shown (see [18], [14] for details) that $F_{\theta}$ is a functor and that $F_{\theta} \cong T_{X}$ if $\left(H_{X}, \theta_{X}\right) \cong(H, \theta)$.

\subsection{Smash product and the derived Picard group}

Let $G$ be a group and let $A$ be a k-algebra. Let us fix some map $\Delta: G \rightarrow \operatorname{Aut} A$. We write ${ }^{g} a$ instead of $\Delta(g)(a)$ for $g \in G, a \in A$.

Definition 3. The smash product of $A$ and $\mathbf{k} G$ is the algebra $A \# G$ whose elements are the pairs $a \# g(a \in A, g \in G)$ with multiplication defined by the formula

$$
(a \# g)(b \# h)=a^{g} b \# g h(a, b \in A, g, h \in G) .
$$

Let $U \in \mathcal{K}_{p}^{\mathrm{b}} A, g \in G$. We define an $A$-complex $U \# g$ in the following way. The set $(U \# g)_{n}$ is formed by the elements $x \# g\left(x \in U_{n}\right)$. The differential $d_{U \# g}$ is defined by the formula $d_{U \# g}(x \# g)=d_{U}(x) \# g(x \in U)$. The $A$-module structure is defined by the formula $(x \# g) a=x^{g} a \# g(x \in U, a \in A)$. For a morphism $f \in \mathcal{C} A(U, V)$ we define a morphism $f \# g \in \mathcal{C} A(U \# g, V \# g)$ by the formula $(f \# g)(x \# g)=f(x) \# g(x \in U)$. We define the $A \# G$-module structure on $U \# G=\oplus_{g \in G} U \# g$ by the formula $(x \# g)(a \# h)=x^{g} a \# g h$ $(a \in U, a \in A, g, h \in G)$. For a morphism $f \in \mathcal{C} A(U, V)$ we define a morphism $f \# G \in$ 
$\mathcal{C}(A \# G)(U \# G, V \# G)$ by the formula $(f \# G)(x \# g)=f(x) \# g(x \in U, g \in G)$. Note that $f_{1} \# G$ is homotopic to $f_{2} \# G$ and $f_{1} \# g$ is homotopic to $f_{2} \# g$ for any $g \in G$ if $f_{1}$ is homotopic to $f_{2}$. So we define $f \# G \in \mathcal{K}_{p}^{\mathrm{b}}(A \# G)(U \# G, V \# G)$ and $f \# g \in \mathcal{K}_{p}^{\mathrm{b}} A(U \# g, V \# g)(g \in G)$ for $f \in \mathcal{K}_{p}^{\mathrm{b}} A(U, V)$. It is easy to see that we have defined a functor $-\# G: \mathcal{K}_{p}^{\mathrm{b}} A \rightarrow \mathcal{K}_{p}^{\mathrm{b}}(A \# G)$. Also for $f \in \mathcal{K}_{p}^{\mathrm{b}} A(U, U \# g)(g \in G)$ we simply write $f^{k}$ instead of $\left(f \# g^{k-1}\right) \ldots(f \# g) f$. Let us define for $g \in G, U \in \mathcal{K}_{p}^{\mathrm{b}} A$ a morphism $s_{g}:(U \# g) \# G \rightarrow U \# G$ by the formula

$$
s_{g}(x \# g \# h)=x \# g h(x \in U, h \in G) .
$$

Note that $s_{g} \circ((f \# g) \# G)=(f \# G) \circ s_{g}$ for $g \in G$ and $f \in \mathcal{K}_{p}^{\mathrm{b}} A(U, V)$.

We recall some results from [14] on the connection between $\operatorname{TrPic}(A)$ and $\operatorname{Tr} \operatorname{Pic}(A \# G)$. We will only need the case $G=C_{t}$ for some $t \in \mathbb{N}$, where by $C_{t}(t \in \mathbb{N} \cup\{\infty\})$ we denote the cyclic group of order $t$. We fix some generating element of $C_{t}$ for each $t$ and denote it by $r$.

A tilting $C_{t}$-functor from $A$ to itself is a triple $(H, \theta, \psi)$, where $(H, \theta)$ is a tilting functor and $\psi \in \mathcal{K}_{p}^{\mathrm{b}} A(H, H \# r)$ is an isomorphism which satisfies the following conditions:

- $\psi \theta\left({ }^{r^{-1}} a\right)=(\theta(a) \# r) \psi$ for any $a \in A$;

- $\psi^{t}=\operatorname{Id}_{H}$ in $\mathcal{K}_{p}^{\mathrm{b}} A$.

Note that $\psi^{k+t}=\psi^{k}$ for any $k \geqslant 0$ by the last condition and so we can define $\psi^{k}$ for any $k \in \mathbb{Z}$. Let now $(H, \theta, \psi)$ be a tilting $C_{t}$-functor from $A$ to $A$. We define an isomorphism $\theta \# \psi: A \# C_{t} \rightarrow \mathcal{K}_{p}^{\mathrm{b}}\left(A \# C_{t}\right)\left(H \# C_{t}, H \# C_{t}\right)$ by the formula

$$
(\theta \# \psi)\left(a \# r^{k}\right)=s_{r^{k}} \circ\left(\psi^{k} \# C_{t}\right) \circ\left(\theta\left(^{r^{-k}} a\right) \# C_{t}\right)(a \in A, k \in \mathbb{Z}) .
$$

Thus, there is a tilting functor $\left(H \# C_{t}, \theta \# \psi\right)$ from $A \# C_{t}$ to itself.

The following lemma follows from [14].

Lemma 1. There is a group $\operatorname{TrPic}_{C_{t}}(A)$ and two homomorphisms $\Phi_{A}: \operatorname{TrPic}_{C_{t}}(A) \rightarrow$ $\operatorname{TrPic}(A)$ and $\Psi_{A}: \operatorname{TrPic}_{C_{t}}(A) \rightarrow \operatorname{TrPic}\left(A \# C_{t}\right)$ which satisfy the following conditions

- for any tilting $C_{t}$-functor $(H, \theta, \psi)$ there is an element $W \in \operatorname{TrPic}_{C_{t}}(A)$ such that $\Phi_{A}(W)=F_{\theta}$ and $\Psi_{A}(W)=F_{\theta \# \psi}$;

- for any $W \in \operatorname{TrPic}_{C_{t}}(A)$ the condition $\Phi_{A}(W) \in \operatorname{Pic}(A)$ is equivalent to the condition $\Psi_{A}(W) \in \operatorname{Pic}\left(A \# C_{t}\right)$.

\subsection{Some groups}

In this section we define some groups which we will use to formulate our main result.

The first group we need is the group of automorphisms of the algebra $\mathbf{k}[x] / x^{N+1}(N \geqslant 1)$. We denote this group by $\mathcal{S}_{N}(\mathbf{k})$. In more detail, $\mathcal{S}_{N}(\mathbf{k})$ is a group whose elements are the sequences $\left(a_{i}\right)_{1 \leqslant i \leqslant N}\left(a_{i} \in \mathbf{k}, a_{1} \neq 0\right)$ and the group operation is defined by the formula

$$
\left(a_{i}\right)_{1 \leqslant i \leqslant n}\left(b_{i}\right)_{1 \leqslant i \leqslant n}=\left(\sum_{j=1}^{i} a_{j} \sum_{k_{1}+\cdots+k_{j}=i} b_{k_{1}} \ldots b_{k_{j}}\right)_{1 \leqslant i \leqslant n} .
$$


In particular, $\mathcal{S}_{1}(\mathbf{k})=\mathbf{k}^{*}$ is the multiplicative group of the field $\mathbf{k}$.

We recall now the definition of braid groups associated to $A_{n}$ and $\widetilde{A}_{n-1}$. These groups are particular cases of a braid group associated to a Coxeter group or a graph but we are going to need only these cases.

Definition 4. The braid group associated to $A_{n}$ is the group $\mathcal{B}\left(A_{n}\right)$ defined by generators $s_{1}, \ldots, s_{n}$ and relations:

$$
s_{i} s_{i+1} s_{i}=s_{i+1} s_{i} s_{i+1} \text { for } 1 \leqslant i \leqslant n-1 \text { and } s_{i} s_{j}=s_{j} s_{i} \text { for } 1 \leqslant i, j \leqslant n,|i-j|>1 .
$$

Definition 5. The braid group associated to $\widetilde{A}_{n-1}$ for $n>2$ is the group $\mathcal{B}\left(\widetilde{A}_{n-1}\right)$ defined by generators $\left\{s_{i}\right\}_{i \in \mathbb{Z}_{n}}$ and relations:

$$
s_{i} s_{i+1} s_{i}=s_{i+1} s_{i} s_{i+1} \text { for } i \in \mathbb{Z}_{n} \text { and } s_{i} s_{j}=s_{j} s_{i} \text { for } i, j \in \mathbb{Z}_{n}, i-j \neq 1,-1 \text {. }
$$

The braid group associated to $\widetilde{A}_{n-1}$ for $n=2$ is the group $\mathcal{B}\left(\widetilde{A}_{1}\right)=F_{2}$, i.e. the free group with two generators.

Now we define the group $\mathcal{A}_{m, n}(m \geqslant 2, n \geqslant 1)$ as the group generated by the elements $s_{1}, \ldots, s_{m}, r_{1}, r_{2}$ satisfying the relations:

$$
\begin{gathered}
s_{i} s_{i+1} s_{i}=s_{i+1} s_{i} s_{i+1} \text { for } 1 \leqslant i \leqslant m-1 \text { and } s_{i} s_{j}=s_{j} s_{i} \text { for } 1 \leqslant i, j \leqslant m,|i-j|>1, \\
s_{i} r_{k}=r_{k} s_{i} \text { for } 1 \leqslant i \leqslant m, k \in\{1,2\}, r_{1} r_{2}=r_{2} r_{1}, r_{2}^{n}=1 \text { and }\left(s_{m} \ldots s_{1}\right)^{m+1}=r_{2}^{m+1} r_{1}^{2 m} .
\end{gathered}
$$

Let $G, H$ be groups and let $\varphi: H \rightarrow \operatorname{Aut}(G)$ be a homomorphism from $H$ to the group of automorphisms of $G$. We denote by $G \rtimes_{\varphi} H$ the semidirect product of $G$ and $H$ such that

$$
\left(g_{1}, h_{1}\right)\left(g_{2}, h_{2}\right)=\left(g_{1} \varphi\left(h_{1}\right)\left(g_{2}\right), h_{1} h_{2}\right)
$$

for $g_{1}, g_{2} \in G, h_{1}, h_{2} \in H$. Let us define the homomorphism $\varphi_{m, n}: C_{n m} \rightarrow \mathcal{B}\left(\widetilde{A}_{m-1}\right)$ by the formula

$$
\varphi_{m, n}\left(r^{l}\right)\left(s_{i}\right)=s_{i+l}\left(i \in \mathbb{Z}_{m}, l \in \mathbb{Z}\right) .
$$

Thus, the group $\mathcal{B}\left(\widetilde{A}_{m-1}\right) \rtimes_{\varphi_{m, n}} C_{n m}$ is defined.

\section{Main result}

Let us consider the algebra $\mathcal{N}_{n m, t m}$ defined in the following way. Let $m, n, t>0$ be some integers. We suppose that $n$ and $t$ are coprime. Let $\mathcal{Q}_{n m}$ be a cyclic quiver with $n m$ vertices, i.e. the quiver whose vertex set is $\mathbb{Z}_{n m}$ and whose arrows are $\beta_{i}: i \rightarrow i+1\left(i \in \mathbb{Z}_{n m}\right)$. Let $\mathcal{I}_{n m, t m}$ be an ideal in the path algebra of $\mathcal{Q}_{n m}$ generated by all paths of length $t m+1$. We denote $\mathcal{N}_{n m, t m}:=\mathbf{k} \mathcal{Q}_{n m} / \mathcal{I}_{n m, t m}$.

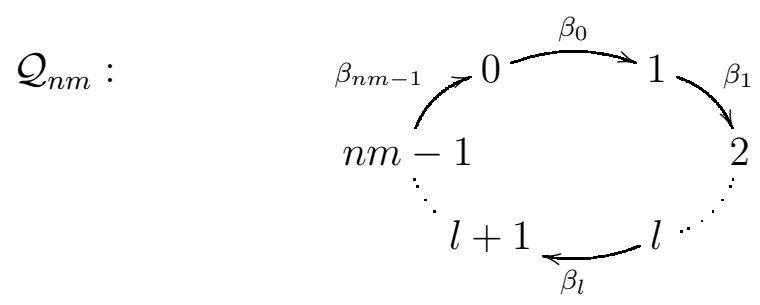

In this work we prove the following theorem. 
Theorem 1. 1) If $m=1$, then

$$
\operatorname{Tr} \operatorname{Pic}\left(\mathcal{N}_{n m, t m}\right)=\operatorname{Tr} \operatorname{Pic}\left(\mathcal{N}_{n, t}\right) \cong C_{n} \times \mathcal{S}_{\left\lfloor\frac{t+n-1}{n}\right\rfloor}(\mathbf{k}) \times C_{\infty}
$$

2) If $m>1$ and $t=1$, then

$$
\operatorname{Tr} \operatorname{Pic}\left(\mathcal{N}_{n m, t m}\right)=\operatorname{Tr} \operatorname{Pic}\left(\mathcal{N}_{n m, m}\right) \cong \mathcal{A}_{m, n} \times \mathbf{k}^{*}
$$

3) If $m>1$ and $t>1$, then

$$
\operatorname{Tr} \operatorname{Pic}\left(\mathcal{N}_{n m, t m}\right) \cong\left(\mathcal{B}\left(\widetilde{A}_{m-1}\right) \rtimes_{\varphi_{m, n}} C_{n m}\right) \times \mathcal{S}_{\left\lfloor\frac{t+n-1}{n}\right\rfloor}(\mathbf{k}) \times C_{\infty}
$$

The proof of the first part of the theorem can be found in Section 3.2, the proof of the second part of the theorem can be found in Section 3.5, the proof of the third part of the theorem can be found in Section 3.8 .

From here on we fix some integers $m, n, t>0$. We omit them in some notation which in fact depends on them. For example, we simply write $\mathcal{N}$ instead of $\mathcal{N}_{n m, t m}$.

\subsection{The description of the Picard group}

Denote by $\operatorname{Pic}(A)$ the Picard group of an algebra $A$, i.e. the group of isomorphism classes of invertible $A^{o p} \otimes A$-modules or equivalently the group of Morita autoequivalences of $A$ modulo natural isomorphisms. If $(H, \theta)$ is a tilting functor from $B$ to $\mathcal{K}_{p}^{\mathrm{b}} A$, the equivalence $F_{\theta}$ is a Morita equivalence iff $H$ is isomorphic to some object $H^{\prime} \in \mathcal{K}_{p}^{\mathrm{b}} A$ concentrated in degree zero ( $H^{\prime}$ is concentrated in degree zero if $H_{i}^{\prime}=0$ for $\left.i \neq 0\right)$. The group $\operatorname{Out}(A)=\operatorname{Aut}(A) / \operatorname{Inn}(A)$ of outer automorphisms of $A$ coincides with $\operatorname{Pic}(A)$ [19] and is clearly a subgroup of $\operatorname{Tr} \operatorname{Pic}(A)$. Every automorphism $\sigma$ of $A$ determines a tilting functor $(A, \bar{\sigma})$, where $\bar{\sigma}(a)\left(a^{\prime}\right)=\sigma(a) a^{\prime}$ for $a, a^{\prime} \in A$. A tilting functor $(A, \bar{\sigma})$ is isomorphic to $\left(A, \bar{\sigma}^{\prime}\right)$ if and only if $\sigma$ coincides with $\sigma^{\prime}$ modulo the subgroup of inner automorphisms. For $\sigma \in \operatorname{Aut}(A)$ we write simply $\sigma$ instead of $F_{\bar{\sigma}}$. Also we denote by $\operatorname{Pic}_{0}(A)$ the subgroup of $\operatorname{Tr} \operatorname{Pic}(A)$ generated by standard equivalences $F_{\theta}$ such that $F_{\theta}(P) \cong P$ in $\mathcal{K}_{p}^{\mathrm{b}} A$ for any $P \in \mathcal{P}_{A}$. Note that $\operatorname{Pic}_{0}(A) \cong \operatorname{Pic}_{0}(\mathcal{N})$ if $A$ is derived equivalent to $\mathcal{N}$, since $\mathcal{N}$ is a uniserial algebra (see [20, Section 4]). Note that if $X, X^{\prime}$ are two two-sided tilting complex of $B^{\mathrm{op}} \otimes A$-modules such that $X \cong X^{\prime}$ in $\mathcal{D}(A)$, then there exists $\sigma \in \operatorname{Pic}(B)$ such that $X \cong \sigma X^{\prime}$ in $\mathcal{D}\left(B^{\mathrm{op}} \otimes A\right)$ [3, Proposition 2.3]. If the action of the equivalences induced by $X, X^{\prime}$ agree on projective modules, then $\sigma \in \operatorname{Pic}_{0}(B)$.

For $i \in \mathbb{Z}_{n m}$ we denote by $e_{i}$ the primitive idempotent of $\mathcal{N}$ corresponding to the vertex $i$ and by $P_{i}$ the projective module $e_{i} \mathcal{N}$. For $w \in e_{j} \mathcal{N} e_{i}$ we denote by $w$ the unique homomorphism from $P_{i}$ to $P_{j}$ which sends $e_{i}$ to $w$. Also we introduce the following auxiliary notation:

$$
\beta_{i, k}=\beta_{i+k-1} \ldots \beta_{i}
$$

In particular, $\beta_{i, 0}=e_{i}$. We write simply $\beta$ instead of $\beta_{i}$ and $\beta^{k}$ instead of $\beta_{i, k}$ if $i$ is clear.

Definition 6. The rotation of $\mathcal{N}$ is an automorphism $\rho \in \operatorname{Aut}(\mathcal{N})$ defined by the formulas $\rho\left(e_{i}\right)=e_{i+1}$ and $\rho\left(\beta_{i}\right)=\beta_{i+1}$. 
Let us consider a sequence $c=\left(c_{i}\right)_{1 \leqslant i \leqslant N}$, where $c_{1}, \ldots, c_{N} \in \mathbf{k}$ and $c_{1} \neq 0$. Let us introduce the notation $N:=\left\lfloor\frac{t+n-1}{n}\right\rfloor, u_{c}:=\sum_{i=1}^{N} c_{i} \beta_{1,(i-1) n m}$. We denote by $\mu_{c} \in \operatorname{Aut}(\mathcal{N})$ the automorphism of $\mathcal{N}$ defined by the formulas

$$
\mu_{c}\left(e_{i}\right)=e_{i}\left(i \in \mathbb{Z}_{n m}\right), \mu_{c}\left(\beta_{i}\right)=\beta_{i}\left(i \in \mathbb{Z}_{n m} \backslash\{0\}\right) \text { and } \mu_{c}\left(\beta_{0}\right)=u_{c} \beta_{0} .
$$

Let us define the map $\xi: C_{n m} \times \mathcal{S}_{N}(\mathbf{k}) \rightarrow \operatorname{Out}(\mathcal{N})$ by the formula $\xi\left(r^{l}, c\right)=\left[\rho^{l} \mu_{c}\right]$, where $[f]$ denotes the class of an automorphism $f$ in $\operatorname{Out}(\mathcal{N})$.

Proposition 1. $\xi$ is an isomorphism of groups.

Proof Let us prove that $\xi$ is a homomorphism. It is not hard to check that $\mu_{c} \mu_{c^{\prime}}=\mu_{c c^{\prime}}$, where the product $c c^{\prime}$ is defined by the formula (2.1). Since $\rho^{n m}=\operatorname{Id}_{\mathcal{N}}$, it remains to prove that $\left[\rho \mu_{c}\right]=\left[\mu_{c} \rho\right]$ for any $c \in \mathcal{S}_{N}(\mathbf{k})$. Let us consider the automorphism $\sigma: \mathcal{N} \rightarrow \mathcal{N}$ defined by the formula $\sigma(x)=\left(1-e_{1}+u_{c}\right)^{-1} x\left(1-e_{1}+u_{c}\right)$ for $x \in \mathcal{N}$. It is easy to check that $\rho \mu_{c}=\sigma \mu_{c} \rho$. Since the automorphism $\sigma$ is inner, we have proved that $\xi$ is a homomorphism.

Let us now prove that $\xi$ is injective. We have to prove that if $\left[\rho^{l} \mu_{c}\right]=\left[\operatorname{Id}_{\mathcal{N}}\right]$, then $n m \mid l$

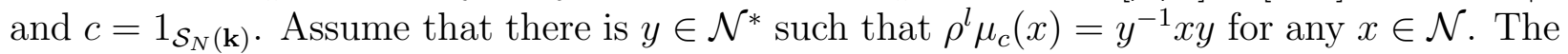
element $y \in \mathcal{N}$ is of the form

$$
y=\sum_{i \in \mathbb{Z}_{n m}}\left(d_{i, 0} e_{i}+d_{i, 1} \beta_{i, 1}+d_{i, 2} \beta_{i, 2}+\cdots+d_{i, t m} \beta_{i, t m}\right),
$$

where $d_{i, 0} \in \mathbf{k}^{*}, d_{i, k} \in \mathbf{k}\left(i \in \mathbb{Z}_{n m}, 1 \leq k \leq t m\right)$. Since $y \rho^{l} \mu_{c}\left(e_{0}\right)=y e_{l}=d_{l, 0} e_{l}+d_{l, 1} \beta_{l, 1}+\ldots$ and $e_{0} y=d_{0,0} e_{0}+d_{n m-1,1} \beta_{n m-1,1}+\ldots$, we have $n m \mid l$ and so $\rho^{l}=\operatorname{Id}_{\mathcal{N}}$.

We deduce from the equalities $y \beta_{i}=\beta_{i} y\left(i \in \mathbb{Z}_{n m} \backslash\{0\}\right)$ that $d_{i+1, k n m}=d_{i, k n m}$ for $i \in \mathbb{Z}_{n m} \backslash\{0\}, 0 \leqslant k \leqslant N-1$. Consequently, $d_{1, k n m}=d_{2, k n m}=\cdots=d_{0, k n m}$ for $0 \leqslant k \leqslant N-1$. We deduce from the last equality and the equality $y u_{c} \beta_{0}=\beta_{0} y$ that

$$
\sum_{i=0}^{k} d_{0, i n m} c_{k-i+1}=d_{0, k n m}(0 \leqslant k \leqslant N-1) .
$$

Since $d_{0,0} \neq 0$, it follows from (3.1) for $k=0$ that $c_{1}=1$. Suppose that we have proved that $c_{1}=1$ and $c_{i}=0$ for $2 \leqslant i \leqslant s(s<N)$. Then it follows from (3.1) for $k=s$ that $c_{s+1}=0$.

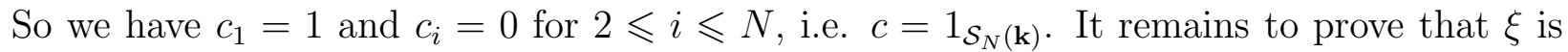
surjective.

If $A$ is a finite-dimensional split algebra over a field, it admits a Wedderbern-Malcev decomposition, i.e. $A=B \oplus J$, where $J$ is the Jacobson radical of $A$, let us fix such a decomposition. Following [21] and [22] we can identify $\operatorname{Out}(A)$ with a group $\widehat{H}_{A} / \widehat{H}_{A} \cap \operatorname{Inn}(A)$, where $\widehat{H}_{A}=\{f \in \operatorname{Aut}(A) \mid f(B) \subseteq B\}$. For $A=\mathcal{N}$ fix $B=\left\langle e_{o}, \ldots e_{n m-1}\right\rangle_{\mathbf{k}}$. Thus any outer automorphism has a representative $f$ such that $f(B) \subseteq B$.

Let us prove that the class in $\operatorname{Out}(\mathcal{N})$ of any automorphism $f \in \widehat{H}_{\mathcal{N}}$ lies in the image of $\xi$. It is easy to see that $f$ is given by the following formulas:

$$
f\left(e_{i}\right)=e_{i+l}, f\left(\beta_{i}\right)=b_{i, 1} \beta_{i+l, 1}+b_{i, 2} \beta_{i+l, n m+1}+\cdots+b_{i, N} \beta_{i+l,(N-1) n m+1},
$$

where $0 \leqslant l<n m, b_{i, 1} \in \mathbf{k}^{*}, b_{i, k} \in \mathbf{k}$ for $i \in \mathbb{Z}_{n m}, 2 \leqslant k \leqslant N$. Applying $\rho^{-l}=\xi\left(r^{-l}\right)$ we can assume that $l=0$. Let us construct by induction an automorphism $g_{k} \in \operatorname{Im} \xi(0 \leqslant k \leqslant n m)$ 
such that $g_{k} f\left(e_{i}\right)=e_{i}\left(i \in \mathbb{Z}_{n m}\right)$ and $g_{k} f\left(\beta_{i}\right)=\beta_{i}(0 \leqslant i<k)$. It is clear that we can take $g_{0}=\operatorname{Id}_{\mathcal{N}}$. Suppose that we have constructed the required automorphism $g_{k}$ for some $0 \leqslant k<n m$. We have

$$
g_{k} f\left(\beta_{k}\right)=a_{k, 1} \beta_{k, 1}+a_{k, 2} \beta_{k, n m+1}+\cdots+a_{k, N} \beta_{i,(N-1) n m+1}=\rho^{k}\left(u_{a_{k}}\right) \beta_{k}
$$

for some $a_{k}=\left(a_{k, i}\right)_{1 \leqslant i \leqslant N} \in \mathcal{S}_{N}(\mathbf{k})$. In this case we set $g_{k+1}=\left(\rho^{k} \mu_{a_{k}}^{-1} \rho^{-k}\right) g_{k}$. It is easy to check that $g_{k+1}$ satisfies the required conditions. Then $g_{n m} f=\operatorname{Id}_{\mathcal{N}}$, i.e. $f=g_{n m}^{-1} \in \operatorname{Im} \xi$.

Corollary 1. $\operatorname{Pic}_{0}(\mathcal{N}) \cong \mathcal{S}_{N}(\mathbf{k})$.

\subsection{Generating set for $\operatorname{Tr} \operatorname{Pic}(\mathcal{N})$}

Let $\mathcal{N}$ be as above. If $U$ is a module, then we also denote by $U$ the corresponding complex concentrated in degree 0 . When we write $\cdots \rightarrow \underline{U} \rightarrow \ldots$ we mean the complex whose zero component is $U$. If $V, V^{\prime}$ are complexes, then by $(\ldots, \underline{f}, \ldots)$ we denote the morphism of complexes whose zero component is $f: V_{0} \rightarrow V_{0}^{\prime}$. We simply write $f$ instead of $(\ldots, 0, \underline{f}, 0, \ldots)$. For $i \in \mathbb{Z}_{n m}, 1 \leqslant k \leqslant m-1$ we introduce the complexes

$$
X_{i}:=P_{i-t m} \stackrel{\beta}{\rightarrow} P_{i-t m+1} \stackrel{\beta^{t m}}{\rightarrow} \underline{P_{i+1}} \text { and } Y_{i, k}:=\underline{P_{i}} \stackrel{\beta^{k}}{\rightarrow} P_{i+k} .
$$

If $m>1$, then for $l \in \mathbb{Z}_{m}$ we introduce the $\mathcal{N}$-complex

$$
H_{l}=\left(\bigoplus_{i \in \mathbb{Z}_{n m}, m \nmid i-l} P_{i}\right) \oplus\left(\bigoplus_{i \in \mathbb{Z}_{n m}, m \mid i-l} X_{i}\right) .
$$

In this case we define an equivalence $S_{\theta_{l}}: \mathcal{P}_{\mathcal{N}} \rightarrow \operatorname{add} H_{l}$ in the following way:

$$
\begin{aligned}
& S_{\theta_{l}}\left(P_{i}\right)= \begin{cases}P_{i}, & \text { if } m \nmid i-l \text { and } m \nmid i-1-l, \\
P_{i+1}, & \text { if } m \mid i-l, \\
X_{i-1}, & \text { if } m \mid i-1-l,\end{cases} \\
& S_{\theta_{l}}\left(\beta_{i}\right)= \begin{cases}\beta_{i}, & \text { if } m \nmid i-l+1 \text { and } m \nmid i-1, \\
\beta_{i+1} \beta_{i}, & \text { if } m \mid i-l+1, \\
\operatorname{Id}_{P_{i+1},}, & \text { if } m \mid i-l .\end{cases}
\end{aligned}
$$

If $m>1$ and $t=1$, then for $l \in \mathbb{Z}_{m}$ we introduce the $\mathcal{N}$-complex

$$
Q_{l}=\bigoplus_{i \in \mathbb{Z}_{n m}, m \mid i-l}\left(P_{i} \oplus \bigoplus_{k=1}^{m-1} Y_{i, k}\right)
$$

In this case we define an equivalence $S_{\varepsilon_{l}}: \mathcal{P}_{\mathcal{N}} \rightarrow \operatorname{add} Q_{l}$ in the following way:

$$
S_{\varepsilon_{l}}\left(P_{i}\right)= \begin{cases}P_{i-m}, & \text { if } m \mid i-l, \\ Y_{i+k-m, m-k}, & \text { if } m \mid i+k-l \text { for some } k, 1 \leqslant k \leqslant m-1,\end{cases}
$$




$$
S_{\varepsilon_{l}}\left(\beta_{i}\right)= \begin{cases}\beta_{i-m, m}, & \text { if } m \mid i-l, \\ \operatorname{Id}_{P_{i-m+1}}, & \text { if } m \mid i-l+1, \\ \left.\underline{\left(\operatorname{Id}_{P_{i+k-m}}\right.}, \beta_{i}\right), & \text { if } m \mid i-l+k \text { for some } k, 2 \leqslant k \leqslant m-1 .\end{cases}
$$

For simplicity of notation we write $\mathcal{H}_{l}$ instead of $F_{\theta_{l}}$ and $\mathcal{Q}_{l}$ instead of $F_{\varepsilon_{l}}$.

The following theorem was proved in [14].

Theorem 2. 1) If $m=1$, then $\operatorname{Tr} \operatorname{Pic}(\mathcal{N})$ is generated by the shift and $\operatorname{Pic}(\mathcal{N})$;

2) If $m>1, t>1$, then $\operatorname{Tr} \operatorname{Pic}(\mathcal{N})$ is generated by the shift, $\operatorname{Pic}(\mathcal{N})$ and $\mathcal{H}_{l}\left(l \in \mathbb{Z}_{m}\right)$;

3) If $m>1, t=1$, then $\operatorname{Tr} \operatorname{Pic}(\mathcal{N})$ is generated by the shift, $\operatorname{Pic}(\mathcal{N}), \mathcal{H}_{l}$ and $\mathcal{Q}_{l}\left(l \in \mathbb{Z}_{m}\right)$.

Proof of part 1 of Theorem 1 Let $m=1$. By part 1 of Theorem 2 we have $\operatorname{TrPic}(\mathcal{N})=$ $\langle\operatorname{Pic}(\mathcal{N}), G\rangle$, where $G \cong C_{\infty}$ is the cyclic group generated by the shift. By Proposition 1 we have $\operatorname{Pic}(\mathcal{N}) \cong C_{n} \times \mathcal{S}_{\left\lfloor\frac{t+n-1}{n}\right\rfloor}(\mathbf{k})$. It is clear that $\operatorname{Pic}(\mathcal{N}) \cap G=\left\{\operatorname{Id}_{\mathcal{K}_{p}^{\text {b }} \mathcal{N}}\right\}$. Since the shift commutes with any element of $\operatorname{Pic}(\mathcal{N})$, we have

$$
\operatorname{Tr} \operatorname{Pic}(\mathcal{N})=\operatorname{Pic}(\mathcal{N}) \times G \cong C_{n} \times \mathcal{S}_{\left\lfloor\frac{t+n-1}{n}\right\rfloor}(\mathbf{k}) \times C_{\infty}
$$

From here on we assume that $m \geqslant 2$. We need the following result (which is similar to [9, Proposition 3]).

Lemma 2. There is a homomorphism $\eta: \mathcal{B}\left(\widetilde{A}_{m-1}\right) \rightarrow \operatorname{TrPic}(\mathcal{N})$ which sends $s_{i}$ to $\mathcal{H}_{i}$ for all $i \in \mathbb{Z}_{m}$.

Proof If $m=2$, then $\mathcal{B}\left(\widetilde{A}_{m-1}\right)=F_{2}$ and there is nothing to check. Assume that $m>2$. We need to check that $\mathcal{H}_{i}$ satisfy the relations of the braid group associated to $\widetilde{A}_{m-1}$. The relations $\mathcal{H}_{i} \mathcal{H}_{j}=\mathcal{H}_{j} \mathcal{H}_{i}$ for $|i-j|>1$ are straightforward. Let us check that $\mathcal{H}_{l} \mathcal{H}_{l+1} \mathcal{H}_{l}=$ $\mathcal{H}_{l+1} \mathcal{H}_{l} \mathcal{H}_{l+1}$. For simplicity let us assume that $m>3$, the case $m=3$ is similar. The diagram below shows how $\mathcal{H}_{l}, \mathcal{H}_{l+1} \mathcal{H}_{l}$ and $\mathcal{H}_{l} \mathcal{H}_{l+1} \mathcal{H}_{l}$ act on the projective modules and morphisms between them. In this computation we can take $\tau_{i}=0, i>1$. The morphism $\mathcal{H}_{l+1} \mathcal{H}_{l}\left(\beta_{k m+l+1}\right)$ can be computed using the construction from Section 2.2 with $\alpha_{1}=-\mathrm{Id}$, $\alpha_{2}=-\beta_{k m+l}$, in all other cases $\alpha_{i}=0, i>0$. The action of $\mathcal{H}_{l} \mathcal{H}_{l+1} \mathcal{H}_{l}$ on the indecomposable projective modules and morphisms between them not appearing in this diagram is trivial.

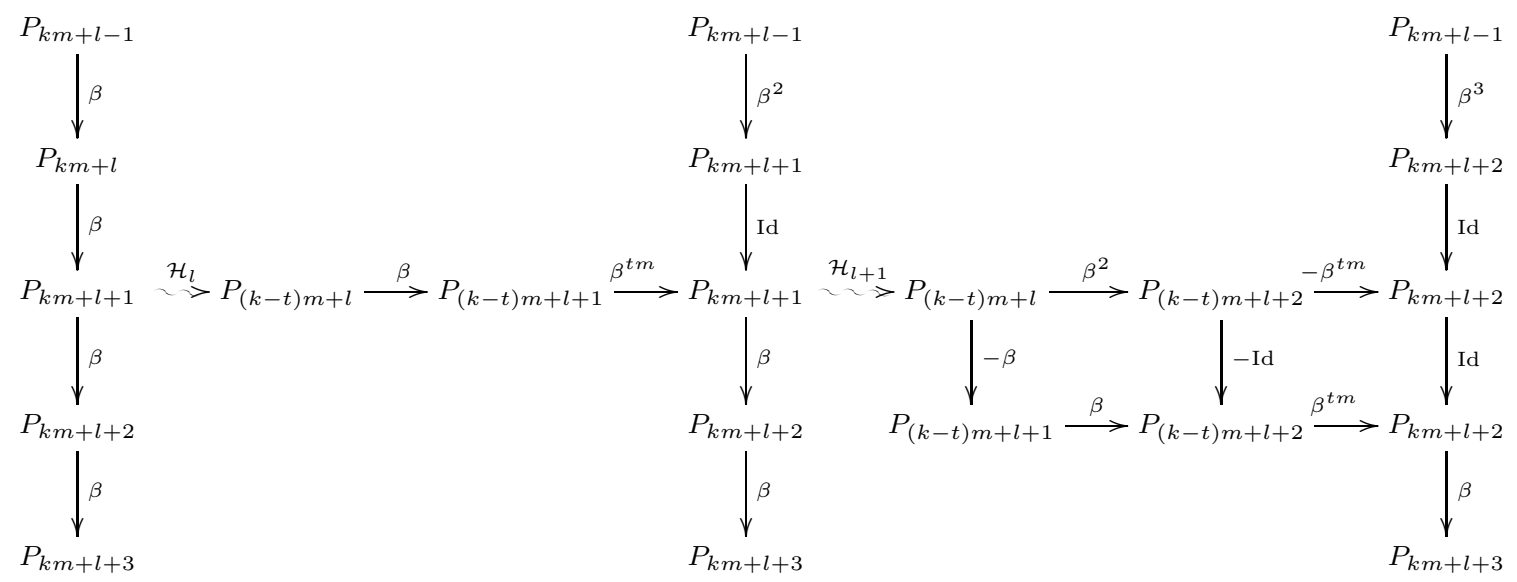




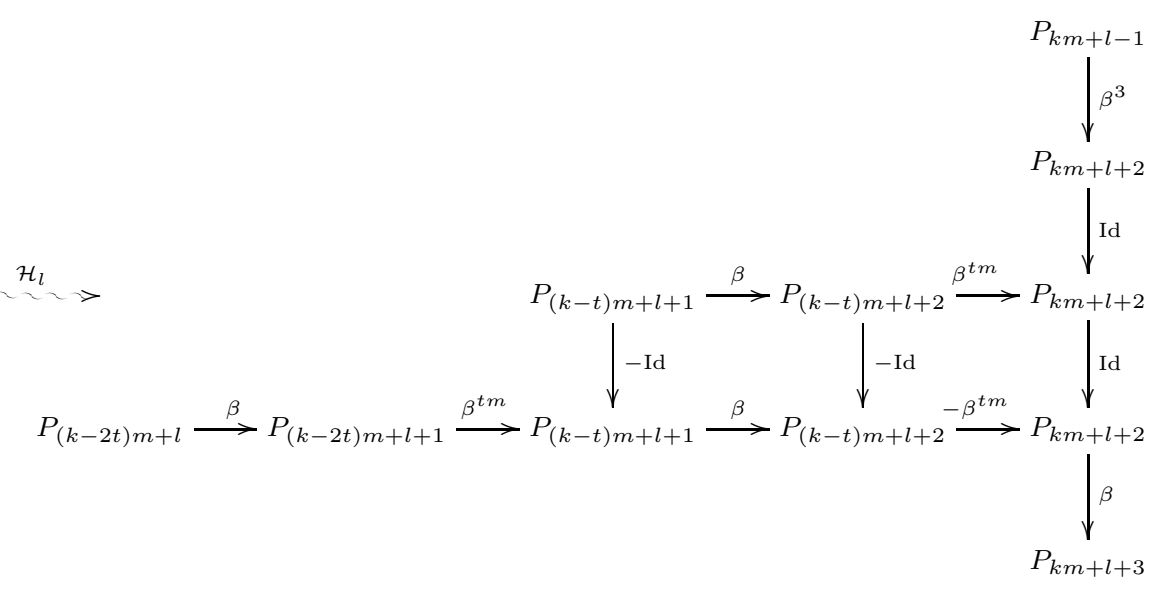

Let us compute $\mathcal{H}_{l} \mathcal{H}_{l+1}$.

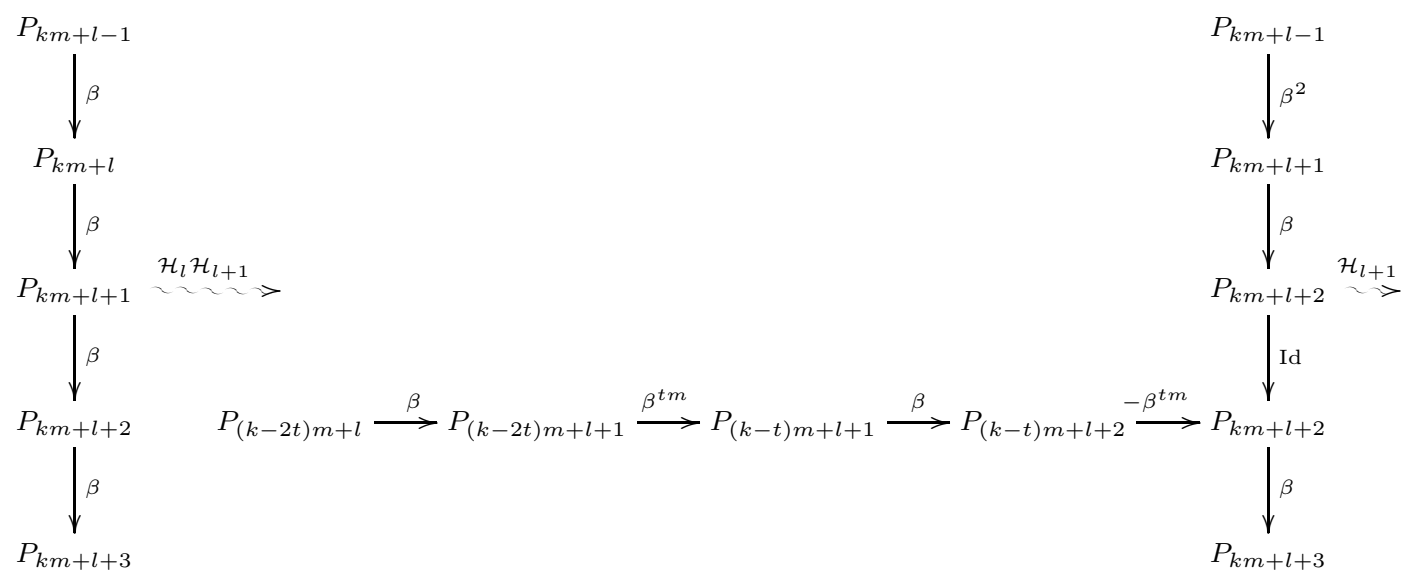

$\mathcal{H}_{l+1} \mathcal{H}_{l} \mathcal{H}_{l+1}\left(P_{k m+l+2}\right)$ is the totalization of the following bicomplex, where $\tau_{2}$ and $\tau_{3}$ are constructed according to the algorithm from Section 2.2 and are shown as dotted lines.

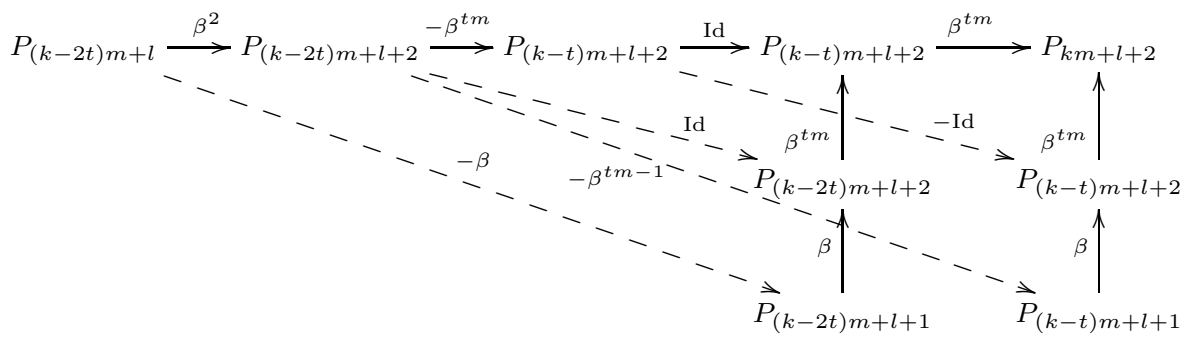

$$
\begin{aligned}
& \mathcal{H}_{l+1} \mathcal{H}_{l} \mathcal{H}_{l+1}\left(P_{k m+l+2}\right)=P_{(k-2 t) m+l} \stackrel{\left(\begin{array}{c}
\beta^{2} \\
-\beta
\end{array}\right)}{\longrightarrow} P_{(k-2 t) m+l+2} \oplus P_{(k-2 t) m+l+1} \stackrel{\left(\begin{array}{cc}
-\beta^{t m} & 0 \\
\text { Id } & \beta \\
-\beta^{t m-1} & 0
\end{array}\right)}{\longrightarrow} \\
& P_{(k-t) m+l+2} \oplus P_{(k-2 t) m+l+2} \oplus P_{(k-t) m+l+1} \stackrel{\left(\begin{array}{ccc}
\mathrm{Id} & \beta^{t m} & 0 \\
-\mathrm{Id} & 0 & \beta
\end{array}\right)}{\longrightarrow} P_{(k-t) m+l+2} \oplus P_{(k-t) m+l+2} \stackrel{\left(\beta^{t m} \beta^{t m}\right)}{\longrightarrow} P_{k m+l+2}
\end{aligned}
$$

The isomorphism from $\mathcal{H}_{l+1} \mathcal{H}_{l} \mathcal{H}_{l+1}\left(P_{k m+l+2}\right)$ to $\mathcal{H}_{l} \mathcal{H}_{l+1} \mathcal{H}_{l}\left(P_{k m+l+2}\right)$ is given by

$$
f=\left(\mathrm{Id},(0-\mathrm{Id}),\left(0-\beta^{t m-1}-\mathrm{Id}\right),(-\mathrm{Id}-\mathrm{Id}), \underline{\mathrm{Id}}\right)
$$


with the inverse

$$
f^{-1}=\left(\mathrm{Id},\left(\begin{array}{c}
\beta \\
-\mathrm{Id}
\end{array}\right),\left(\begin{array}{c}
0 \\
0 \\
-\mathrm{Id}
\end{array}\right),\left(\begin{array}{c}
0 \\
-\mathrm{Id}
\end{array}\right), \underline{\mathrm{Id}}\right) .
$$

Indeed, it is easy to see that $\mathcal{H}_{l} \mathcal{H}_{l+1} \mathcal{H}_{l}\left(P_{k m+l+2}\right)$ is a direct summand of $\mathcal{H}_{l+1} \mathcal{H}_{l} \mathcal{H}_{l+1}\left(P_{k m+l+2}\right)$, since $\mathcal{H}_{l+1} \mathcal{H}_{l} \mathcal{H}_{l+1}\left(P_{k m+l+2}\right)$ is an image of an indecomposable object under an equivalence, the other direct summand is isomorphic to zero. And we get:

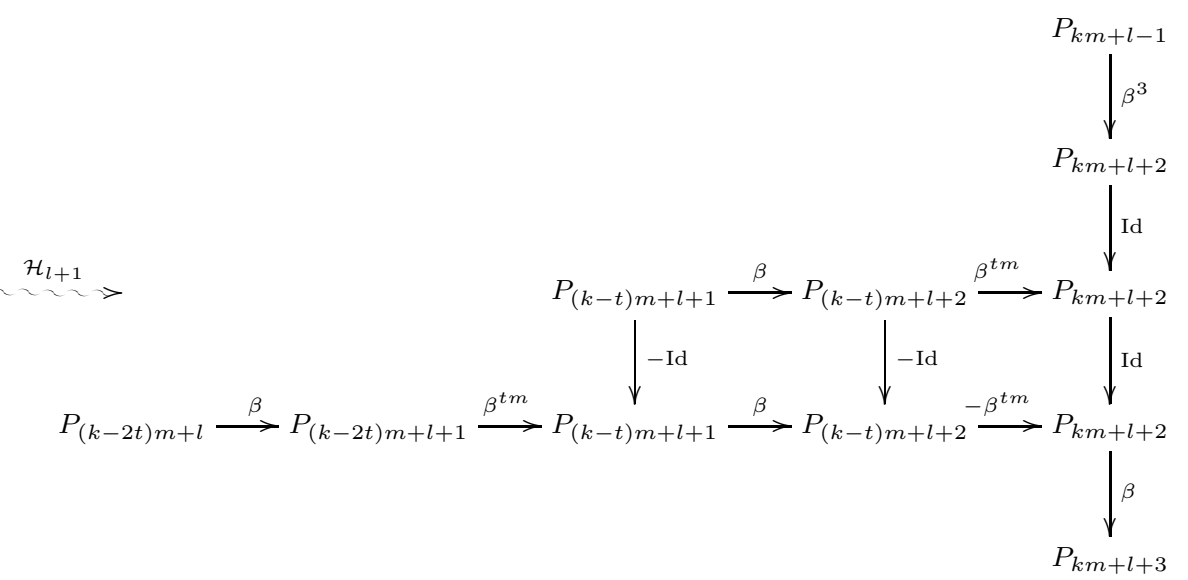

Here we replace $\mathcal{H}_{l+1} \mathcal{H}_{l} \mathcal{H}_{l+1}\left(\beta_{k m+l+1}\right)$ and $\mathcal{H}_{l+1} \mathcal{H}_{l} \mathcal{H}_{l+1}\left(\beta_{k m+l+2}\right)$ by $f \mathcal{H}_{l+1} \mathcal{H}_{l} \mathcal{H}_{l+1}\left(\beta_{k m+l+1}\right)$ and $\mathcal{H}_{l+1} \mathcal{H}_{l} \mathcal{H}_{l+1}\left(\beta_{k m+l+2}\right) f^{-1}$ respectively.

\subsection{The algebra $\mathcal{R}$}

In this and the next sections we assume that $t=1$. Let us consider an algebra $\mathcal{R}$ defined as a path algebra of a quiver with relations. Let $\mathcal{Q}_{m, n}$ be a quiver whose set of vertices is $\{1, \ldots, m\} \times \mathbb{Z}_{n}$. The set of arrows of $\mathcal{Q}_{m, n}$ is:

$$
\gamma_{i, j}:(i, j) \rightarrow(i+1, j) \text { and } \gamma_{i, j}^{\prime}:(i+1, j) \rightarrow(i, j+1)\left(1 \leqslant i \leqslant m-1, j \in \mathbb{Z}_{n}\right) .
$$

Let us define the ideal of relations $\mathcal{I}_{m, n}$. If $m>2$, then it is generated by the elements $\gamma_{i+1, j} \gamma_{i, j}, \gamma_{i, j+1}^{\prime} \gamma_{i+1, j}^{\prime}$ and $\gamma_{i, j+1} \gamma_{i, j}^{\prime}-\gamma_{i+1, j}^{\prime} \gamma_{i+1, j}$ for $1 \leqslant i \leqslant m-2, j \in \mathbb{Z}_{n}$. If $m=2$, then the ideal $\mathcal{I}_{m, n}$ is generated by the elements $\gamma_{1, j+1} \gamma_{1, j}^{\prime} \gamma_{1, j}$ and $\gamma_{1, j+1}^{\prime} \gamma_{1, j+1} \gamma_{1, j}^{\prime}$ for $j \in \mathbb{Z}_{n}$. We define the algebra $\mathcal{R}$ by the formula $\mathcal{R}=\mathrm{k} \mathcal{Q}_{m, n} / \mathcal{I}_{m, n}$. We omit the indices of $\gamma$ and $\gamma^{\prime}$ if they can be easily recovered from the context. It is known that the algebra $\mathcal{R}$ is derived equivalent to the algebra $\mathcal{N}$.

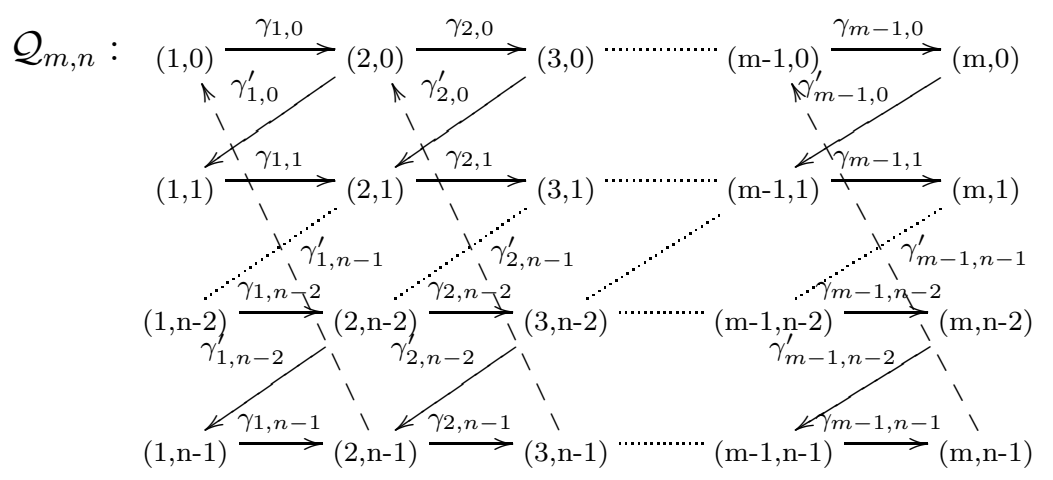


We denote by $e_{i, j}$ the primitive idempotent corresponding to the vertex $(i, j)$. By $P_{i, j}$ we denote the projective $\mathcal{R}$-module $e_{i, j} \mathcal{R}$ and by $P_{\left[i_{1}, j_{1}\right]\left[i_{2}, j_{2}\right]}$ the projective $\mathcal{R}^{\text {op }} \otimes \mathcal{R}$-module $\mathcal{R} e_{i_{1}, j_{1}} \otimes e_{i_{2}, j_{2}} \mathcal{R}$. Also we denote by $\tau$ the automorphism of the algebra $\mathcal{R}$ defined by the formulas

$$
\tau\left(e_{i, j}\right)=e_{i, j+1}, \tau\left(\gamma_{k, j}\right)=\gamma_{k, j+1}, \tau\left(\gamma_{k, j}^{\prime}\right)=\gamma_{k, j+1}^{\prime}\left(1 \leqslant i \leqslant m, 1 \leqslant k \leqslant m-1, j \in \mathbb{Z}_{n}\right) .
$$

Note that $\tau^{-1}$ is a Nakayama automorphism of $\mathcal{R}$ and so it commutes with any standard equivalence by [16, Proposition 5.2].

Let us consider the following $\mathcal{R}^{\mathrm{op}} \otimes \mathcal{R}$-complexes

$$
T_{i}=\left(\bigoplus_{j \in \mathbb{Z}_{n}} P_{[i, j][i, j]} \stackrel{\mu_{i}}{\rightarrow} \underline{\mathcal{R}}\right)
$$

where $\mu_{i}(a \otimes b)=a b$ for $a \otimes b \in P_{[i, j][i, j]}$. We denote by $\mathcal{T}_{i}$ the functor $-\otimes_{\mathcal{R}} T_{i}$.

The algebra $\mathcal{R}$ with an additional grading was studied in [15] (there $\mathcal{R}$ is denoted by $\left.A_{m, 0}^{n}\right)$. The arguments from [15] can be applied to our case to obtain the following result:

Proposition 2. 1) $\mathcal{T}_{i}$ is an equivalence. In particular, $T_{i}$ is a two-sided tilting complex for $\mathcal{R}$.

2) There is an injective homomorphism of groups $\lambda: \mathcal{B}\left(A_{m}\right) \rightarrow \operatorname{Tr} \operatorname{Pic}(\mathcal{R})$ such that $\lambda\left(s_{i}\right)=\mathcal{T}_{i}$ $(1 \leqslant i \leqslant m)$.

In particular, there is an $A_{m}$ configuration of 0 -spherical sequences of length $n$ in the category $\mathcal{K}_{p}^{\mathrm{b}} \mathcal{R}$. For more details on spherical twists see [10].

\subsection{Construction of an isomorphism from $\operatorname{Tr} \operatorname{Pic}(\mathcal{R})$ to $\operatorname{Tr} \operatorname{Pic}(\mathcal{N})$}

In this section we construct explicitly the isomorphism $\operatorname{Tr} \operatorname{Pic}(\mathcal{R}) \cong \operatorname{Tr} \operatorname{Pic}(\mathcal{N})$.

Let us consider the following $\mathcal{R}$-complexes:

$$
U_{i, j}=\left(\underline{P_{1, j}} \stackrel{\gamma}{\rightarrow} P_{2, j} \stackrel{\gamma}{\rightarrow} \ldots \stackrel{\gamma}{\rightarrow} P_{i, j}\right)
$$

It can be easily verified that $U:=\oplus_{1 \leqslant i \leqslant m, j \in \mathbb{Z}_{n}} U_{i, j}$ is a tilting complex for $\mathcal{R}$. For $i \in \mathbb{Z}_{n m}$ we denote by $p(i)$ and $q(i)$ the unique integers such that $0 \leqslant p(i) \leqslant m-1,0 \leqslant q(i) \leqslant n-1$ and $p(i)+m q(i)=i$ in $\mathbb{Z}_{n m}$. Let us define an equivalence $S_{\omega}: \mathcal{P}_{A} \rightarrow \operatorname{add} U$ by the formulas

$$
S_{\omega}\left(P_{i}\right)=U_{m-p(i), q(i)}, S_{\omega}\left(\beta_{i}\right)= \begin{cases}\left(\operatorname{Id}_{P_{1, q(i)}}, \ldots, \operatorname{Id}_{P_{m-p(i)-1}, q(i)}\right), & \text { if } p(i) \leqslant m-2, \\ \gamma_{1, q(i)}^{\prime} \gamma_{1, q(i)}, & \text { if } p(i)=m-1 .\end{cases}
$$

The tilting functor $(U, \omega)$ defines an isomorphism of groups

$$
L_{\omega}: \operatorname{Tr} \operatorname{Pic}(\mathcal{R}) \rightarrow \operatorname{Tr} \operatorname{Pic}(\mathcal{N})
$$

by the formula $L_{\omega}(F)=\bar{F}_{\omega} F F_{\omega}$, where $\bar{F}_{\omega}$ is the quasi-inverse equivalence for $F_{\omega}$. By [20] $L_{\omega}\left(\operatorname{Pic}_{0}(\mathcal{R})\right)=\operatorname{Pic}_{0}(\mathcal{N})$ and $L_{\omega}([1])=[1]$. It is also easy to see that $L_{\omega}(\tau)=\rho^{m}$. 
Lemma 3. For any projective $\mathcal{N}$-module $P$ we have

$$
L_{\omega}\left(\mathcal{T}_{i}\right)(P) \cong \begin{cases}\mathcal{H}_{m-i}(P), & \text { if } i>1, \\ \mathcal{Q}_{m-1}(P)[1], & \text { if } i=1 .\end{cases}
$$

Proof Let us consider the case $i>1$. We have to prove that $F_{\omega} \mathcal{H}_{m-i}\left(P_{j}\right) \cong \mathcal{T}_{i} F_{\omega}\left(P_{j}\right)$ for any $j \in \mathbb{Z}_{n m}$. Let $p(j)$ and $q(j)$ be as above. Note that $2 \leqslant p(j)+i \leqslant 2 m-1$. Then we have to prove that

$$
F_{\omega} \mathcal{H}_{m-i}\left(P_{p(j)+m q(j)}\right) \cong \mathcal{T}_{i}\left(U_{m-p(j), q(j)}\right) .
$$

If $p(j)+i \notin\{m, m+1\}$, then by definition $F_{\omega} \mathcal{H}_{m-i}\left(P_{p(j)+m q(j)}\right)=U_{m-p(j), q(j)}$. If $m-p(j)<$ $i-1$, then it is clear that $\mathcal{T}_{i}\left(U_{m-p(j), q(j)}\right) \cong U_{m-p(j), q(j)}$. If $m-p(j)>i$, then $\mathcal{T}_{i}\left(U_{m-p(j), q(j)}\right)$ is isomorphic to the cone of a map from

$$
C:=\left(\underline{P_{i, q(j)-1}} \stackrel{\operatorname{Id}_{P_{i, q(j)-1}}}{\longrightarrow} P_{i, q(j)-1}\right)[2-i] \oplus\left(\underline{P_{i, q(j)}} \stackrel{\operatorname{Id}_{P_{i, q(j)}}}{\longrightarrow} P_{i, q(j)}\right)[1-i]
$$

to $U_{m-p(j), q(j)}$. Since $C \cong 0, \mathcal{T}_{i}\left(U_{m-p(j), q(j)}\right) \cong U_{m-p(j), q(j)}$ in $\mathcal{K}_{p}^{\mathrm{b}} \mathcal{R}$. It remains to consider the cases $p(j)=m-i$ and $p(j)=m-i+1$.

Assume that $p(j)=m-i$. In this case $F_{\omega} \mathcal{H}_{m-i}\left(P_{p(j)+m q(j)}\right)=F_{\omega}\left(P_{p(j)+1+m q(j)}\right)=$ $U_{i-1, q(j)}$. At the same time $\mathcal{T}_{i}\left(U_{m-p(j), q(j)}\right)$ is isomorphic to the cone of the map

$$
\left.\underline{\left(\gamma_{i-1, q(j)-1}^{\prime}\right.}, \gamma_{i, q(j)-1}^{\prime} \gamma_{i, q(j)-1}\right)[2-i] \oplus \operatorname{Id}_{P_{i, q(j)}}[1-i]
$$

from

$$
C:=\left(\underline{P_{i, q(j)-1}} \stackrel{\operatorname{Id}_{P_{i, q(j)-1}}}{\longrightarrow} P_{i, q(j)-1}\right)[2-i] \oplus P_{i, q(j)}[1-i]
$$

to $U_{i, q(j)}$. It is easy to see that this cone is isomorphic to $U_{i-1, q(j)}$.

Let us now consider the case $p(j)=m-i+1$. In this case

$$
F_{\omega} \mathcal{H}_{m-i}\left(P_{p(j)+m q(j)}\right)=F_{\omega}\left(X_{p(j)+m q(j)-1}\right) .
$$

Note that $F_{\omega}\left(\beta_{p(j)+m(q(j)-1), m}\right)$ is homotopic to $\left(\gamma_{i-1, q(j)-1}^{\prime} \gamma_{i-1, q(j)-1}\right)[2-i]$. Applying $F_{\omega}$ to $X_{i+m q(j)}$ we obtain the totalization of the following bicomplex

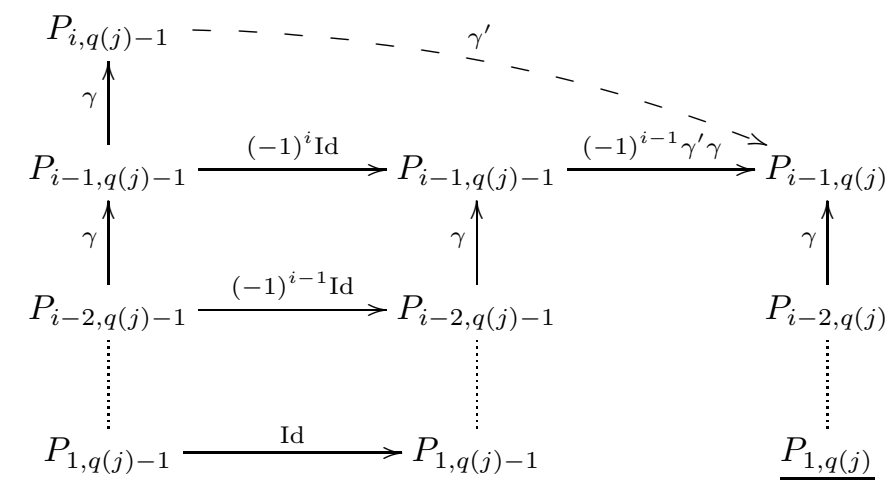

The dotted arrow was constructed according to the algorithm from Section 2.2. It is clear that the totalization is isomorphic to the cone of

$$
P_{i, q(j)-1}[2-i] \stackrel{\gamma_{i-1, q(j)-1}^{\prime}[2-i]}{\longrightarrow} U_{i-1, q(j)}
$$

and it is easy to see that $\mathcal{T}_{i}\left(U_{m-p(j), q(j)}\right)$ is isomorphic to the same cone. The case $i=1$ is analogous and so it is left to the reader. 
To prove part 2 of Theorem 1 we also need the following lemma.

Lemma 4. $\mathcal{Q}_{m-1}^{2} \mathcal{H}_{m-2} \ldots \mathcal{H}_{0}\left(P_{i}\right) \cong P_{i-m-1}$ for all $i \in \mathbb{Z}_{n m}$.

Proof If $m \mid i$, then

$$
\mathcal{Q}_{m-1}^{2} \mathcal{H}_{m-2} \ldots \mathcal{H}_{0}\left(P_{i}\right)=\mathcal{Q}_{m-1}^{2} \mathcal{H}_{m-2} \ldots \mathcal{H}_{1}\left(P_{i+1}\right)=\cdots=\mathcal{Q}_{m-1}^{2}\left(P_{i+m-1}\right)=P_{i-m-1} .
$$

Now let $m \mid i-k$ where $1 \leqslant k \leqslant m-1$. We have

$$
\begin{aligned}
& \mathcal{Q}_{m-1}^{2} \mathcal{H}_{m-2} \ldots \mathcal{H}_{0}\left(P_{i}\right)=\mathcal{Q}_{m-1}^{2} \mathcal{H}_{m-2} \ldots \mathcal{H}_{k-1}\left(P_{i}\right)=\mathcal{Q}_{m-1}^{2} \mathcal{H}_{m-2} \ldots \mathcal{H}_{k}\left(X_{i-1}\right) \\
= & \mathcal{Q}_{m-1}^{2} \mathcal{H}_{m-2} \ldots \mathcal{H}_{k+1}\left(P_{i-m-1} \stackrel{\beta^{2}}{\rightarrow} P_{i-m+1} \stackrel{\beta^{m}}{\longrightarrow} \underline{P_{i+1}}\right) \\
= & \cdots=\mathcal{Q}_{m-1}^{2}\left(P_{i-m-1} \stackrel{\beta^{m-k}}{\longrightarrow} P_{i-k-1} \stackrel{\beta^{m}}{\longrightarrow} \underline{P_{i-k-1+m}}\right) .
\end{aligned}
$$

$\mathcal{Q}_{m-1}\left(P_{i-m-1} \stackrel{\beta^{m-k}}{\longrightarrow} P_{i-k-1} \stackrel{\beta^{m}}{\longrightarrow} \underline{P_{i-k-1+m}}\right)$ is the totalization of the bicomplex

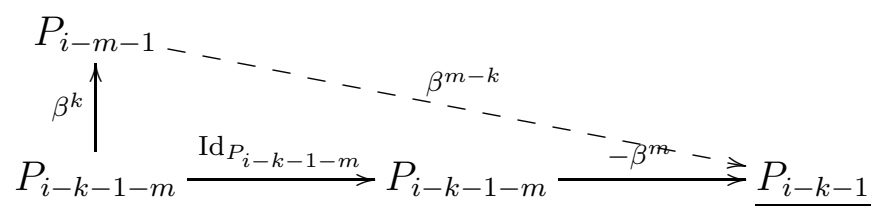

So

$$
\mathcal{Q}_{m-1}^{2} \mathcal{H}_{m-2} \ldots \mathcal{H}_{0}\left(P_{i}\right) \cong \mathcal{Q}_{m-1}\left(P_{i-m-1} \stackrel{\beta^{m-k}}{\longrightarrow} \underline{P_{i-k-1}}\right) \cong P_{i-m-1}
$$

\subsection{The case $t=1$}

In this section we prove part 2 of Theorem 1. So we assume that $t=1$. Since $\mathcal{R}$ is derived equivalent to $\mathcal{N}$, it follows from Corollary 1 that there is some isomorphism $\zeta_{0}: \mathbf{k}^{*} \rightarrow$ $\operatorname{Pic}_{0}(\mathcal{R})$. First, we prove the following proposition.

Proposition 3. There is a monomorphism of groups

$$
\zeta: \mathcal{A}_{m, n} \times \mathbf{k}^{*} \rightarrow \operatorname{Tr} \operatorname{Pic}(\mathcal{R})
$$

such that:

$$
\zeta(1, a)=\zeta_{0}(a)\left(a \in \mathbf{k}^{*}\right), \zeta\left(s_{i}, 1\right)=\mathcal{T}_{i}(1 \leqslant i \leqslant m), \zeta\left(r_{1}, 1\right)=[1] \text { and } \zeta\left(r_{2}, 1\right)=\tau .
$$

Proof To prove the existence of $\zeta$ we have to check that

1) every element of $\operatorname{Pic}_{0}(\mathcal{R})$ commutes with $\mathcal{T}_{i}(1 \leqslant i \leqslant m)$, [1] and $\tau$;

2) [1] and $\tau$ commute with $\mathcal{T}_{i}(1 \leqslant i \leqslant m)$ and with each other;

3) $\mathcal{T}_{i} \mathcal{T}_{j}=\mathcal{T}_{j} \mathcal{T}_{i}$ if $1 \leqslant i, j \leqslant m$ and $|i-j|>1$;

4) $\mathcal{T}_{i} \mathcal{T}_{i+1} \mathcal{T}_{i}=\mathcal{T}_{i+1} \mathcal{T}_{i} \mathcal{T}_{i+1}$ for $1 \leqslant i \leqslant m-1$; 
5) $\left(\mathcal{T}_{m} \ldots \mathcal{T}_{1}\right)^{m+1}=\tau^{m+1}[2 m]$;

6) $\tau^{n}=\operatorname{Id}_{\mathcal{R}}$.

Any element of $\operatorname{Pic}_{0}(\mathcal{R})$ is of the form $-\otimes_{\mathcal{R}} \mathcal{R}_{\sigma}$ for some $\sigma \in \operatorname{Aut}(\mathcal{R})$ such that $\sigma\left(e_{i, j}\right)=$ $e_{i, j}$ for all $1 \leqslant i \leqslant m, j \in \mathbb{Z}_{n}$ (here $\mathcal{R}_{\sigma}$ is an $\mathcal{R}$-bimodule which coincides with $\mathcal{R}$ as a left module and has the right multiplication $*$ defined by the formula $a * b=a \sigma(b)$ for $\left.a \in \mathcal{R}_{\sigma}, b \in \mathcal{R}\right)$. The map $\psi: \mathcal{R} \otimes_{\mathcal{R}} \mathcal{R}_{\sigma} \rightarrow \mathcal{R}_{\sigma} \otimes_{\mathcal{R}} \mathcal{R}$ defined by the formula $\psi(a \otimes b)=$ $a \otimes \sigma^{-1}(b)$ is an isomorphism of bimodules. Also for all $1 \leqslant i \leqslant m$ and $j \in \mathbb{Z}_{n}$ we have an isomorphism of bimodules $\psi_{i, j}: P_{[i, j],[i, j]} \otimes_{\mathcal{R}} \mathcal{R}_{\sigma} \rightarrow \mathcal{R}_{\sigma} \otimes_{\mathcal{R}} P_{[i, j],[i, j]}$ defined by the formula $\psi_{i, j}(a \otimes b \otimes c)=a \otimes e_{i, j} \otimes \sigma^{-1}(b c)$. Then the map $\left(\oplus_{j \in \mathbb{Z}_{n}} \psi_{i, j}, \underline{\psi}\right): T_{i} \otimes_{\mathcal{R}} \mathcal{R}_{\sigma} \rightarrow \mathcal{R}_{\sigma} \otimes_{\mathcal{R}} T_{i}$ is an isomorphism of complexes of $\mathcal{R}$-bimodules. It is clear that the shift commutes with any standard equivalence. As was mentioned above $\tau$ commutes with any standard equivalence too. So 1) and 2) hold. 3) and 4) follow from Proposition 2 and 6) is clear.

By [15, Lemma 4.30] (applied to our case) we have

$$
\left(\mathcal{T}_{m} \ldots \mathcal{T}_{1}\right)^{m+1}\left(P_{i, j}\right)=P_{i, j+m+1}[2 m] .
$$

So the element $\left(\mathcal{T}_{m} \ldots \mathcal{T}_{1}\right)^{m+1}[-2 m] \tau^{-(m+1)}$ lies in $\operatorname{Pic}_{0}(\mathcal{R})$. There is a canonical map from $\operatorname{Tr} \operatorname{Pic}(\mathcal{R})$ to the group of isomorphism classes of $\mathcal{R}$-bimodules inducing stable autoequivalences of $\mathcal{R}$ (see [3, Section 3.4] for details). This map is injective on $\operatorname{Pic}_{0}(\mathcal{R})$. At the same time it sends $\mathcal{T}_{i}$ to $\mathcal{R}$ and $[-2 m]$ to $\Omega_{\mathcal{R}^{\mathrm{op}} \otimes \mathcal{R}}^{2 m}(\mathcal{R})$. So it is enough to prove that $\Omega_{\mathcal{R}^{\mathrm{oP}} \otimes \mathcal{R}}^{2 m}(\mathcal{R})$ is isomorphic to $\mathcal{R}_{\tau^{m+1}}$. From [23, Section 4] it follows that $\Omega_{\mathcal{N}^{\mathrm{op} \otimes \mathcal{N}}}^{2 m}(\mathcal{N}) \cong \mathcal{N}_{\rho^{m(m+1)}}$. There are $\mathcal{N}$ - $\mathcal{R}$-bimodule $M$ and $\mathcal{R}-\mathcal{N}$-bimodule $\bar{M}$ which correspond to $F_{\omega}$ and $\bar{F}_{\omega}$ respectively (see [3, Corollary 2.15]). Then we have

$$
\Omega_{\mathcal{R}^{\mathrm{op} \otimes \mathcal{R}}}^{2 m}(\mathcal{R}) \cong \bar{M} \otimes_{\mathcal{N}} \Omega_{\mathcal{N}^{\mathrm{op}} \otimes \mathcal{N}}^{2 m}(\mathcal{N}) \otimes_{\mathcal{N}} M \cong \bar{M} \otimes_{\mathcal{N}} \mathcal{N}_{\rho^{m(m+1)}} \otimes_{\mathcal{N}} M \cong \mathcal{R}_{\tau^{m+1}}
$$

in the stable category of $\mathcal{R}$-bimodules. Here we use the fact that $\rho^{-m}$ is a Nakayama automorphism and so $\rho^{m(m+1)} F_{\omega} \cong F_{\omega} \tau^{m+1}$ by [16, Proposition 5.2].

Let us now consider an element $\left(s r_{1}^{-l_{1}} r_{2}^{-l_{2}}, a\right) \in \mathcal{A}_{m, n} \times \mathbf{k}^{*}$, where $s \in \mathcal{B}\left(A_{n}\right)$. Suppose

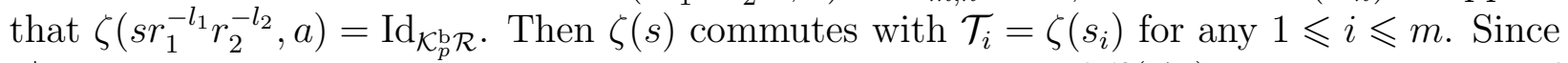
$\left.\zeta\right|_{\mathcal{B}\left(A_{n}\right)}$ is injective by Proposition 2, $s$ belongs to the center of $\mathcal{B}\left(A_{n}\right)$. Then by results of [24] we have $s=\left(s_{n} \ldots s_{1}\right)^{k(m+1)}$ for some $k \in \mathbb{Z}$. So $\left(\mathcal{T}_{m} \ldots \mathcal{T}_{1}\right)^{k(m+1)}=\zeta_{0}(a) \tau^{l_{2}}\left[l_{1}\right]$. Then it follows from (3.2) that $l_{1}=2 m k$ and $n \mid l_{2}-k(m+1)$. Thus, $s r_{1}^{-l_{1}} r_{2}^{-l_{2}}=1, \zeta_{0}(a)=1$. Since $\zeta_{0}: \mathbf{k}^{*} \rightarrow \operatorname{Pic}_{0}(\mathcal{R})$ is an isomorphism, $a=1$ and so $\left(s r_{1}^{-l_{1}} r_{2}^{-l_{2}}, a\right)=(1,1)$. Consequently, $\zeta$ is a monomorphism.

Proof of part 2 of Theorem 1 By Proposition 3 it is enough to prove that $L_{\omega}\left(\mathcal{T}_{i}\right)(1 \leqslant$ $i \leqslant m), L_{\omega}\left(\operatorname{Pic}_{0}(\mathcal{R})\right), L_{\omega}(\tau)$ and [1] generate $\operatorname{Tr} \operatorname{Pic}(\mathcal{N})$. Denote by $X$ the subgroup of $\operatorname{TrPic}(\mathcal{N})$ generated by these elements. $\operatorname{Pic}_{0}(\mathcal{N})$ belongs to $X$. By Lemma $3 \mathcal{H}_{i}(0 \leqslant i \leqslant$ $m-2), \mathcal{Q}_{m-1}$ belong to $X$. By Lemma $4 \rho^{-(m+1)}$ belongs to $X$, thus by Proposition 1 and since $\rho^{m} \in X$, the subgroup $\operatorname{Pic}(\mathcal{N})$ also belongs to $X$. Since

$$
\mathcal{H}_{m-1}=\rho \mathcal{H}_{m-2} \rho^{-1} \text { and } \mathcal{Q}_{i}=\rho^{i+1-m} \mathcal{Q}_{m-1} \rho^{m-1-i}(0 \leqslant i \leqslant m-2),
$$

$\mathcal{H}_{m-1}$ and $\mathcal{Q}_{i}(0 \leqslant i \leqslant m-2)$ belong to $X$. Thus by part 3 of Theorem 2 we have $X=\operatorname{Tr} \operatorname{Pic}(\mathcal{N})$. 
From here on we assume that $t \geqslant 2$. Let us denote $\mathcal{N}(n t m, t m)$ by $\tilde{\mathcal{N}}$. Moreover we denote by $\widetilde{\mathcal{H}}_{l}, \widetilde{\mathcal{Q}}_{l}: \mathcal{K}_{p}^{\mathrm{b}} \widetilde{\mathcal{N}} \rightarrow \mathcal{K}_{p}^{\mathrm{b}} \widetilde{\mathcal{N}}\left(l \in \mathbb{Z}_{t m}\right)$ the standard equivalences whose construction for $\widetilde{\mathcal{N}}$ coincides with that of $\mathcal{H}_{l}$ and $\mathcal{Q}_{l}\left(l \in \mathbb{Z}_{m}\right)$ for $\mathcal{N}$ respectively. Similarly, we define $\widetilde{e}_{i}, \widetilde{\beta}_{i}$, $\widetilde{P}_{i}\left(i \in \mathbb{Z}_{n t m}\right), \widetilde{X}_{i}, \widetilde{Y}_{i}\left(i \in \mathbb{Z}_{t m}\right)$.

\subsection{Some properties of $\mathcal{B}\left(\widetilde{A}_{N-1}\right)$}

In this section we list some properties of the group $\mathcal{B}\left(\widetilde{A}_{N-1}\right)$. Most of them are known but we collect them here for convenience. Let us introduce the notation $\Pi:=s_{N} \ldots s_{1}$. The following result can be found in [25]:

Lemma 5. The group $\mathcal{B}\left(\widetilde{A}_{N-1}\right)$ is torsion-free and its center is trivial.

The next fact can be found in [12, Section 5.1]:

Lemma 6. There is a monomorphism of groups $\phi_{N}: \mathcal{B}\left(\widetilde{A}_{N-1}\right) \rightarrow \mathcal{B}\left(A_{N}\right)$ such that

$$
\phi_{N}\left(s_{i}\right)=s_{i+1}(0 \leqslant i \leqslant N-2) \text { and } \phi_{N}\left(s_{N-1}\right)=s_{N} \Pi s_{1} \Pi^{-1} s_{N}^{-1} .
$$

Corollary 2. There is a monomorphism of groups $\widetilde{\eta}: \mathcal{B}\left(\widetilde{A}_{t m-1}\right) \rightarrow \operatorname{Tr} \operatorname{Pic}(\tilde{\mathcal{N}})$ such that $\widetilde{\eta}\left(s_{i}\right)=\widetilde{\mathcal{H}}_{i}$ for $i \in \mathbb{Z}_{t m}$.

Proof By Proposition 3 there is a monomorphism of groups $\eta^{\prime}: \mathcal{B}\left(A_{t m}\right) \rightarrow \operatorname{Tr} \operatorname{Pic}(\widetilde{\mathcal{N}})$ which sends $s_{i}$ to $L_{\omega}\left(\mathcal{T}_{t m-i+1}\right)$. Let us compute $\eta^{\prime} \phi_{t m}\left(s_{i}\right)$. By Lemma 3 we have

$$
\begin{aligned}
\widetilde{\mathcal{H}}_{i} & =L_{\omega}\left(\mathcal{T}_{t m-i}\right) W_{i}=\eta^{\prime}\left(s_{i+1}\right) W_{i}(0 \leqslant i \leqslant t m-2), \\
\widetilde{\mathcal{Q}}_{t m-1} & =L_{\omega}\left(\mathcal{T}_{1}\right)[-1] W_{t m-1}=\eta^{\prime}\left(s_{t m}\right)[-1] W_{t m-1}
\end{aligned}
$$

for some $W_{i} \in \operatorname{Pic}_{0}(\tilde{\mathcal{N}})$, for $i \in \mathbb{Z}_{t m}$. By Lemma 4 we have

$$
\widetilde{\mathcal{Q}}_{t m-1}^{2} \widetilde{\mathcal{H}}_{t m-2} \ldots \widetilde{\mathcal{H}}_{0}=\rho^{-(t m+1)} W
$$

for some $W \in \operatorname{Pic}_{0}(\tilde{\mathcal{N}})$. Since $\operatorname{Pic}_{0}(\tilde{\mathcal{N}})$ lies in the center of $\operatorname{Tr} \operatorname{Pic}(\tilde{\mathcal{N}})$, we have

$$
\eta^{\prime}\left(s_{t m} \Pi s_{1} \Pi^{-1} s_{t m}^{-1}\right) W_{0}=\rho^{-(t m+1)} \widetilde{\mathcal{H}}_{0} \rho^{t m+1}=\widetilde{\mathcal{H}}_{t m-1} .
$$

By Lemma 2 there is a homomorphism $\widetilde{\eta}$ with the required properties. If $\widetilde{\eta}(s)=\operatorname{Id}_{\mathcal{K}_{p}^{\mathrm{b}} \widetilde{\mathcal{N}}}$

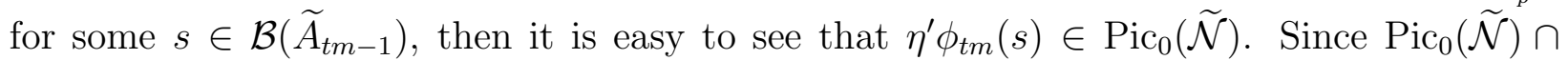
$\operatorname{Im} \eta^{\prime}=\left\{\operatorname{Id}_{\mathcal{K}_{p}^{\mathrm{b}} \tilde{\mathcal{N}}}\right\}$ (this follows, for example, from Proposition 3 ) and the maps $\eta^{\prime}$ and $\phi_{\text {tm }}$ are monomorphic by Lemma [6, we have $s=1$. Consequently, $\widetilde{\eta}$ is a monomorphism.

Lemma 7 . There is a monomorphism of groups $\psi: \mathcal{B}\left(\widetilde{A}_{m-1}\right) \rightarrow \mathcal{B}\left(\widetilde{A}_{t m-1}\right)$ defined by the formula

$$
\psi\left(s_{i}\right)=s_{i} s_{i+m} \ldots s_{i+(t-1) m}\left(i \in \mathbb{Z}_{m}\right) .
$$


Proof It is easy to check that $\psi$ is a homomorphism of groups. We only need to check that it is monomorphic.

We are going to consider $\mathcal{B}\left(\widetilde{A}_{m-1}\right)$ as a subgroup of the braid group of a two-dimensional surface. Let $L$ be the complex plane without the origin $\mathbb{C}^{*}$, or equivalently a sphere with two punctures, denote by $\Delta_{m}=\left\{\left(x_{0}, \ldots, x_{m-1}\right) \in L^{m} \mid x_{i}=x_{j}\right.$ for some $\left.i \neq j\right\}$. The symmetric group $S_{m}$ acts on $L^{m}$, the action is free on $L^{m} \backslash \Delta_{m}$. Consider $X_{m}=\left(L^{m} \backslash \Delta_{m}\right) / S_{m}$, fix a base point $\left(b_{0}, \ldots, b_{m-1}\right) \in X_{m}$, then the $m$-strand braid group of $L$ is defined to be the fundamental group of $X_{m}$, we will denote it by $B r_{m}(L)$. Each element of $B r_{m}(L)$ may be represented by a set of paths $\gamma_{0}, \ldots, \gamma_{m-1}:[0,1] \rightarrow L$ such that there is a permutation $\pi$ of $\{0, \ldots, m-1\}$ such that $\gamma_{i}(0)=b_{i}, \gamma_{i}(1)=b_{\pi(i)}$ and for each $t \in[0,1]$ the points $\gamma_{i}(t)$ are all distinct. Then $\mathcal{B}\left(\widetilde{A}_{m-1}\right)$ is a normal subgroup in $B r_{m}(L)$ with the quotient isomorphic to $\mathbb{Z}$. A good choice for a set of basepoints for $m$-strand braids in $\mathbb{C}^{*}$ is the set of $m$-th roots of unity. Then the standard generators $s_{i}$ correspond to the standard braid generators $s_{i}^{\prime}$, which exchange two adjacent basepoints in the simplest possible way 26.

Consider a $t$-sheeted covering of $L$ with the covering space $\tilde{L}$ homeomorphic to $L, p$ : $\tilde{L} \rightarrow L$. If we identify $L$ with $\mathbb{C}^{*}$ this covering can be given by $z^{t}$. Consider also the group $\operatorname{Br}_{t m}(\tilde{L})$. The base point $\left(\tilde{b}_{0}, \ldots, \tilde{b}_{t m-1}\right) \in \tilde{X}_{t m}$ is chosen in such a way that $p\left(\tilde{b}_{i+s m}\right)=b_{i}$, $s=0, \ldots, t-1$. This covering induces a covering $\tilde{L} \backslash\left\{\tilde{b}_{0}, \ldots, \tilde{b}_{t m-1}\right\} \rightarrow L \backslash\left\{b_{0}, \ldots, b_{m-1}\right\}$, which will be also denoted by $p$. There is a lift of $s_{i}^{\prime}$ from $L$ to $\tilde{L}$, it will be denoted by $\tilde{s}_{i}^{\prime}$. The element $\tilde{s}_{i}^{\prime}$ exchanges $\tilde{b}_{i+s m}$ and $\tilde{b}_{i+1+s m}$ for $s=0, \ldots, t-1$. The homomorphism $\psi$ is consistent with the lift $\tilde{s}_{i}^{\prime}$ of $s_{i}^{\prime}$.

The fundamental group $\pi_{1}\left(\tilde{L} \backslash\left\{\tilde{b}_{0}, \ldots, \tilde{b}_{t m-1}\right\}\right)$ is isomorphic to $F_{t m+1}$. Since $p$ is a covering it induces a monomorphism

$$
p_{*}: \pi_{1}\left(\tilde{L} \backslash\left\{\tilde{b}_{0}, \ldots, \tilde{b}_{t m-1}\right\}\right) \simeq F_{t m+1} \rightarrow \pi_{1}\left(L \backslash\left\{b_{0}, \ldots, b_{m-1}\right\}\right) \simeq F_{m+1} .
$$

The index of $F_{t m+1}$ in $F_{m \pm 1}$ is equal to $t$.

Let us check that $\mathcal{B}\left(\widetilde{A}_{m-1}\right)$ acts faithfully on $\pi_{1}\left(L \backslash\left\{b_{0}, \ldots, b_{m-1}\right\}\right) \simeq F_{m+1}$. Let us consider the mapping class group $M C G_{m}(L)$ of $L \backslash\left\{b_{0}, \ldots, b_{m-1}\right\}$, i.e. the group of all path components in the space of all homeomorphisms from $L$ to $L$ which keep $\left\{b_{0}, \ldots, b_{m-1}\right\}$ fixed as a set. By [27] there is a long exact sequence

$$
\cdots \rightarrow \pi_{1}(\operatorname{Homeo}(L)) \rightarrow B r_{m}(L) \stackrel{w}{\rightarrow} M C G_{m}(L) \rightarrow \pi_{0}(\operatorname{Homeo}(L)) \rightarrow 1,
$$

where $\operatorname{Homeo}(L)$ is the space of all homeomorphisms from $L$ to $L$. The group $\pi_{1}($ Homeo $(L))$ is isomorphic to $\mathbb{Z}$, its image in $B r_{m}(L)$ is generated by the central element of $B r_{m}(L)$, corresponding to the rotation of $\mathbb{C}^{*}$ by $2 \pi$. Thus the intersection of $\mathcal{B}\left(\widetilde{A}_{m-1}\right)$ and the kernel of $w$ is trivial and $w$ induces an embedding from $\mathcal{B}\left(\widetilde{A}_{m-1}\right)$ to $M C G_{m}(L)$. One can consider the stabilizer of one puncture in $M C G_{m}(L)$, if we identify $L$ with $\mathbb{C}^{*}$ it would be the stabilizer of the origin, this stabilizer is a subgroup of the mapping class group $M C G_{m+1}(\mathbb{C})$ of all homeomorphisms $\mathbb{C} \rightarrow \mathbb{C}$ which keep $\left\{b_{0}, \ldots, b_{m-1}, 0\right\}$ fixed as a set. It is easy to see that $\mathcal{B}\left(\widetilde{A}_{m-1}\right)$ stabilizes the puncture, thus it can be embedded into $M C G_{m+1}(\mathbb{C})$. It is well known that $M C G_{m+1}(\mathbb{C})$ coincides with the $m+1$-strand braid group of $\mathbb{C}$ or equivalently a sphere with one puncture and that this group acts faithfully on $\pi_{1}\left(L \backslash\left\{b_{0}, \ldots, b_{m-1}\right\}\right) \simeq F_{m+1}$. Thus $\mathcal{B}\left(\widetilde{A}_{m-1}\right)$ is embedded into a group which acts faithfully on $\pi_{1}\left(L \backslash\left\{b_{0}, \ldots, b_{m-1}\right\}\right) \simeq F_{m+1}$, hence it acts faithfully on $\pi_{1}\left(L \backslash\left\{b_{0}, \ldots, b_{m-1}\right\}\right) \simeq F_{m+1}$. 
If we denote by $y_{i}$ the loop around $b_{i}$ and by $y$ the loop around the origin, then the action of $\mathcal{B}\left(\widetilde{A}_{m-1}\right)$ is defined as follows:

$$
s_{i}\left(y_{i}\right)=y_{i+1}, s_{i}\left(y_{i+1}\right)=y_{i+1}^{-1} y_{i} y_{i+1}, s_{i}\left(y_{j}\right)=y_{j}(j \neq i, i+1), s_{i}(y)=y .
$$

Similarly, $\mathcal{B}\left(\widetilde{A}_{t m-1}\right)$ acts faithfully on $\pi_{1}\left(\tilde{L} \backslash\left\{\tilde{b}_{0}, \ldots, \tilde{b}_{t m-1}\right\}\right) \simeq F_{t m+1}$ and these two actions are compatible, in particular, $p_{*} \psi(g)(\gamma)$ is homotopic to $g\left(p_{*}(\gamma)\right)$, for $g \in \mathcal{B}\left(\widetilde{A}_{m-1}\right)$, $\gamma \in \pi_{1}\left(\tilde{L} \backslash\left\{\tilde{b}_{0}, \ldots, \tilde{b}_{t m-1}\right\}\right)$, this can be easily checked on the basis.

Assume that $\psi$ is not monomorphic, i.e. there exists $g \neq 1 \in \mathcal{B}\left(\widetilde{A}_{m-1}\right)$ such that $\psi(g)=1$. Since $g \neq 1$ and the action of $\mathcal{B}\left(\widetilde{A}_{m-1}\right)$ on $F_{m+1}$ is faithful $g(\gamma) \neq \gamma$ for some $\gamma \in F_{m+1}$, but $\gamma^{t} \in p_{*}\left(F_{t m+1}\right)$, hence $\gamma^{t}=p_{*}\left(\gamma^{\prime}\right)$ for some $\gamma^{\prime} \in F_{t m+1}$ and

$$
g(\gamma)^{t}=g\left(\gamma^{t}\right)=g\left(p_{*}\left(\gamma^{\prime}\right)\right)=p_{*}\left(\psi(g)\left(\gamma^{\prime}\right)\right)=p_{*}\left(\gamma^{\prime}\right)=\gamma^{t}
$$

Finally, $g(\gamma) \neq \gamma$ and $g(\gamma)^{t}=\gamma^{t}$, which is not possible in a free group [28, Chapter I, Propositions 2.16, 2.17].

It follows from Corollary 2 and Lemma 7 that there is a monomorphism of groups $\bar{\eta}=\widetilde{\eta} \psi$ : $\mathcal{B}\left(\widetilde{A}_{m-1}\right) \rightarrow \operatorname{Tr} \operatorname{Pic}(\widetilde{\mathcal{N}})$ which sends $s_{i} \in \mathcal{B}\left(\widetilde{A}_{m-1}\right)(0 \leqslant i \leqslant m-1)$ to $\mathcal{H}_{i} \mathcal{H}_{i+m} \ldots \mathcal{H}_{i+(t-1) m}$.

\subsection{Faithful action of $\mathcal{B}\left(\widetilde{A}_{m-1}\right)$}

Let us consider the homomorphism $\Delta: C_{t} \rightarrow \operatorname{Aut}(\widetilde{\mathcal{N}})$ defined by the formula $\Delta(r)=\widetilde{\rho}^{n m}$ (where $\widetilde{\rho}$ is the rotation of $\widetilde{\mathcal{N}}$ ).

For $l \in \mathbb{Z}_{m}$ let us introduce the $\tilde{\mathcal{N}}$-complexes

$$
\bar{H}_{l}=\left(\bigoplus_{i \in \mathbb{Z}_{n t m}, m \nmid i-l} \widetilde{P}_{i}\right) \oplus\left(\bigoplus_{i \in \mathbb{Z}_{n t m}, m \mid i-l} \tilde{X}_{i}\right) .
$$

In this case we define an equivalence $S_{\bar{\theta}_{l}}: \mathcal{P}_{\widetilde{\mathcal{N}}} \rightarrow \operatorname{add} \bar{H}_{l}$ in the following way:

$$
\begin{aligned}
& S_{\bar{\theta}_{l}}\left(\widetilde{P}_{i}\right)= \begin{cases}\widetilde{P}_{i}, & \text { if } m \nmid i-l \text { and } m \nmid i-1-l, \\
\widetilde{P}_{i+1}, & \text { if } m \mid i-l, \\
\widetilde{X}_{i-1}, & \text { if } m \mid i-1-l,\end{cases} \\
& S_{\bar{\theta}_{l}}\left(\widetilde{\beta}_{i}\right)= \begin{cases}\widetilde{\beta}_{i}, & \text { if } m \nmid i-l+1 \text { and } m \nmid i-1, \\
\widetilde{\beta}_{i+1} \widetilde{\beta}_{i}, & \text { if } m \mid i-l+1, \\
\operatorname{Id}_{\widetilde{P}_{i+1},}, & \text { if } m \mid i-l .\end{cases}
\end{aligned}
$$

It is clear that $F_{\bar{\theta}_{l}}=\widetilde{\mathcal{H}}_{l} \widetilde{\mathcal{H}}_{l+m} \ldots \widetilde{\mathcal{H}}_{l+(t-1) m}$. Note that for any $i \in \mathbb{Z}_{n t m}$ there is an isomorphism $f_{i}: \widetilde{P}_{i} \rightarrow \widetilde{P}_{i+n m} \# r$ defined by the equality $f_{i}\left(\widetilde{e}_{i}\right)=\widetilde{e}_{i+n m} \# r$. We define $s_{i, l}: S_{\bar{\theta}_{l}}\left(\widetilde{P}_{i}\right) \rightarrow S_{\bar{\theta}_{l}}\left(\widetilde{P}_{i+n m}\right) \# r$ in the following way. If $S_{\bar{\theta}_{l}}\left(\widetilde{P}_{i}\right)=\widetilde{P}_{j}$ for some $j$, then $s_{i, l}=f_{j}$. If $S_{\bar{\theta}_{l}}\left(\widetilde{P}_{i}\right)=\widetilde{X}_{i}$, then $s_{i, l}=\left(f_{i-t m-1}, f_{i-t m}, \underline{f_{i}}\right)$. Let $\pi_{i, l}: \bar{H}_{l} \rightarrow S_{\bar{\theta}_{l}}\left(\widetilde{P}_{i}\right)$ and $\iota_{i, l}: S_{\bar{\theta}_{l}}\left(\widetilde{P}_{i}\right) \rightarrow \bar{H}_{l}$ 
$\left(i \in \mathbb{Z}_{n t m}\right)$ be the canonical projection and inclusion. We define $\psi_{l} \in \mathcal{K}_{p}^{\mathrm{b}} \mathcal{N}\left(\bar{H}_{l}, \bar{H}_{l} \# r\right)$ by the equality

$$
\psi_{l}=\sum_{i \in \mathbb{Z}_{n t m}}\left(\iota_{i+n m, l} \# r\right) s_{i, l} \pi_{i, l}
$$

Then $\left(\bar{H}_{l}, \bar{\theta}_{l}, \psi_{l}\right)$ is a tilting $C_{t}$-functor from $\tilde{\mathcal{N}}$ to itself.

$\widetilde{\mathcal{N}} \# C_{t}$ is Morita equivalent to $\mathcal{N}$. This equivalence can be constructed in such a way that the induced isomorphism $L: \operatorname{Tr} \operatorname{Pic}(\mathcal{N}) \cong \operatorname{Tr} \operatorname{Pic}\left(\widetilde{\mathcal{N}} \# C_{t}\right)$ satisfies the condition $L\left(\mathcal{H}_{i}\right)=$ $F_{\bar{\theta}_{i} \# \psi_{i}}\left(i \in \mathbb{Z}_{m}\right)$ (see [14, Lemma 3]). It is also clear that $L(\operatorname{Pic}(\mathcal{N}))=\operatorname{Pic}\left(\widetilde{\mathcal{N}} \# C_{t}\right)$.

Proposition 4. The homomorphism $\eta: \mathcal{B}\left(\widetilde{A}_{m-1}\right) \rightarrow \operatorname{Tr} \operatorname{Pic}(\mathcal{N})$ defined in Lemma 0 is injective.

Proof Recall that there is a homomorphism $\bar{\eta}=\widetilde{\eta} \psi: \mathcal{B}\left(\widetilde{A}_{m-1}\right) \rightarrow \operatorname{Tr} \operatorname{Pic}(\widetilde{\mathcal{N}})$ such that $\bar{\eta}\left(s_{i}\right)=F_{\bar{\theta}_{i}}$. The homomorphism $\psi$ defined in Lemma 7 is injective. The homomorphism $\widetilde{\eta}$ is also injective by Corollary 2. Hence $\bar{\eta}$ is injective. By Lemma 1 there are such $W_{i} \in$ $\operatorname{TrPic}_{C_{t}}(\tilde{\mathcal{N}})\left(i \in \mathbb{Z}_{m}\right)$ that $\Phi_{\widetilde{\mathcal{N}}}\left(W_{i}\right)=F_{\bar{\theta}_{i}}$ and $\Psi_{\widetilde{\mathcal{N}}}\left(W_{i}\right)=L\left(\mathcal{H}_{i}\right)$, i.e. $\Phi_{\widetilde{\mathcal{N}}}\left(W_{i}\right)=\bar{\eta}\left(s_{i}\right)$ and $\Psi_{\widetilde{\mathcal{N}}}\left(W_{i}\right)=L \eta\left(s_{i}\right)$. Suppose that $s=s_{i_{1}} \ldots s_{i_{k}} \in \mathcal{B}\left(\widetilde{A}_{m-1}\right)$ is such that $\eta(s)=\operatorname{Id}_{\mathcal{K}_{p}^{\mathrm{b}} \mathcal{N}}$. Then $\Psi_{\widetilde{\mathcal{N}}}\left(W_{i_{1}} \ldots W_{i_{k}}\right)=\operatorname{Id}_{\mathcal{K}_{p}^{\mathrm{b}}\left(\widetilde{\mathcal{N}} \# C_{t}\right)}$ and so

$$
\bar{\eta}(s)=\Phi_{\widetilde{\mathcal{N}}}\left(W_{i_{1}} \ldots W_{i_{k}}\right) \in \operatorname{Pic}(\widetilde{\mathcal{N}})
$$

by Lemma 1. By Proposition 1 we have $\bar{\eta}(s)=\widetilde{\rho}^{l} a$ for some integer number $l$ and $a \in$ $\operatorname{Pic}_{0}(\tilde{\mathcal{N}})$. Then $\bar{\eta}\left(s^{n t m}\right)=a^{n t m}$ lies in the center of $\operatorname{TrPic}(\widetilde{\mathcal{N}})$. Since $a^{n t m} \in \bar{\eta}\left(\mathcal{B}\left(\widetilde{A}_{m-1}\right)\right) \cong$ $\mathcal{B}\left(\widetilde{A}_{m-1}\right)$, we have $\bar{\eta}\left(s^{n t m}\right)=\operatorname{Id}_{\mathcal{K}_{p}^{\mathrm{b}} \widetilde{\mathcal{N}}}$ by Lemma 5. Then $s^{n t m}=1$ and by the same lemma we have $s=1$.

\subsection{The case $t>1$}

In this section we prove the remaining part of Theorem 1 .

Proof of part 3 of Theorem 1 Let us denote $\left\lfloor\frac{t+n-1}{n}\right\rfloor$ by $N$. Let us define a homomorphism $\zeta:\left(\mathcal{B}\left(\widetilde{A}_{m-1}\right) \rtimes_{\varphi_{m, n}} C_{n m}\right) \times \mathcal{S}_{N}(\mathbf{k}) \times C_{\infty} \rightarrow \operatorname{Tr} \operatorname{Pic}(\mathcal{N})$ on the generators:

$$
\begin{aligned}
\zeta\left(s_{i}, 1,1,1\right) & =\mathcal{H}_{i}\left(i \in \mathbb{Z}_{m}\right), \zeta(1, r, 1,1)=\rho, \zeta(1,1,1, r)=[1] \\
\zeta(1,1, c, 1) & =\mu_{c}\left(c \in \mathcal{S}_{N}(\mathbf{k})\right) .
\end{aligned}
$$

Let us prove that $\zeta$ is well defined. Propositions 1 and 4 together with the fact that the shift commutes with any standard equivalence yield that it is enough to prove that

1. $\rho \mathcal{H}_{i} \cong \mathcal{H}_{i+1} \rho\left(i \in \mathbb{Z}_{m}\right)$;

2. $\mu_{c} \mathcal{H}_{i} \cong \mathcal{H}_{i} \mu_{c}\left(i \in \mathbb{Z}_{m}, c \in \mathcal{S}_{N}(\mathbf{k})\right)$. 
The first equality is obvious. Let us prove the second equality. Since $\mu_{c} \mathcal{H}_{i}=\rho^{-i} \mu_{c} \mathcal{H}_{0} \rho^{i}$ and $\mathcal{H}_{i} \mu_{c}=\rho^{-i} \mathcal{H}_{0} \mu_{c} \rho^{i}$, it is enough to prove the second equality for $i=0$.

The equivalence $\mathcal{H}_{0} \mu_{c}$ corresponds to the equivalence $S: \mathcal{P}_{\mathcal{N}} \rightarrow$ add $H_{0}$ which coincides with $S_{\theta_{0}}$ on $P_{i}$ for $i \in \mathbb{Z}_{n m}$ and on $\beta_{i}$ for $i \in \mathbb{Z}_{n m} \backslash\{0\}$ and is defined on $\beta_{0}$ by the formula $S\left(\beta_{0}\right)=u_{c}=S_{\theta_{0}}\left(\beta_{0}\right) u_{c}: P_{1} \rightarrow X_{0}$. On the other hand the equivalence $\mu_{c} \mathcal{H}_{0}$ corresponds to the equivalence $\widetilde{S}: \mathcal{P}_{\mathcal{N}} \rightarrow \operatorname{add} H_{0}$ which coincides with $S_{\theta_{0}}$ on $P_{i}$ for $i \in \mathbb{Z}_{n m}$ and on $\beta_{i}$ for $i \in \mathbb{Z}_{n m} \backslash\{-1\}$ and is defined on $\beta_{-1}$ by the formula $\widetilde{S}\left(\beta_{-1}\right)=u_{c} S_{\theta_{0}}\left(\beta_{-1}\right): P_{-1} \rightarrow P_{1}$. Then the collection of morphisms $\alpha_{i}: S\left(P_{i}\right) \rightarrow \widetilde{S}\left(P_{i}\right)$ defined by the equalities $\alpha_{i}=\operatorname{Id}_{S\left(P_{i}\right)}$ $\left(i \in \mathbb{Z}_{n m} \backslash\{0\}\right)$ and $\alpha_{0}=u_{c}: P_{1} \rightarrow P_{1}$ defines an isomorphism of functors $\alpha: S \rightarrow \widetilde{S}$. So we prove that the definition of $\zeta$ is correct.

It follows from part 2 of Theorem 2 and Proposition 1 that $\zeta$ is surjective. So it remains to prove that it is injective. Assume that $\zeta\left(s, r^{-k_{1}}, c, r^{-k_{2}}\right)=\operatorname{Id}_{\mathcal{K}_{p}^{\mathrm{b}} \mathcal{N}}$. Then

$$
\zeta\left(s^{n m}, 1,1,1\right)=\zeta\left(1,1, c^{-n m}, r^{k_{2} n m}\right) .
$$

Let us denote $\zeta\left(1,1, c^{-n m}, r^{k_{2} n m}\right)$ by $W$. By (3.3) the element W belongs to $G=\zeta\left(\mathcal{B}\left(\widetilde{A}_{m-1}\right)\right)$. On the other hand $W$ commutes with any element of $G$ and $G \cong \mathcal{B}\left(\widetilde{A}_{m-1}\right)$ by Proposition 4 . Then $\zeta(s, 1,1,1)^{n m}=W=\operatorname{Id}_{\mathcal{K}_{p}^{\text {b } \mathcal{N}}}$ by Lemma 5. By the same lemma $\zeta(s, 1,1,1)=\operatorname{Id}_{\mathcal{K}_{p}^{b} \mathcal{N}}$ and so $s=1$ by Proposition 4. It is clear that $\zeta\left(1, r^{-k_{1}}, c, r^{-k_{2}}\right)=\operatorname{Id}_{\mathcal{K}_{p}^{\mathrm{b}} \mathcal{N}}$ iff $n m \mid k_{1}, c=1_{\mathcal{S}_{N}(\mathbf{k})}$ and $k_{2}=0$. Thus, the injectivity of $\zeta$ is proved.

\section{References}

[1] B. Keller, Hochschild cohomology and derived Picard groups. - J. Pure Appl. Algebra 190, 177-196 (2004).

[2] B. Huisgen-Zimmermann, M. Saorín, Geometry of chain complexes and outer automorphisms under derived equivalence. — Trans. Amer. Math. Soc. 353(12), 4757-4777 (2001).

[3] R. Rouquier, A. Zimmermann, Picard groups for derived module categories. - Proc. London Math. Soc. 87(1), 197-225 (2003).

[4] A. Yekutieli, Dualizing complexes, Morita equivalence and the derived Picard group of a ring. - J. London Math. Soc. 60(3), 723-746 (1999).

[5] J. I. Miyachi, A. Yekutieli, Derived Picard groups of finite-dimensional hereditary algebras. - Comp. Math. 129(3), 341-368 (2001).

[6] H. Lenzing, H. Meltzer, The automorphism group of the derived category for a weighted projective line. - Comm. Algebra 28(4), 1685-1700 (2000).

[7] N. Broomhead, D. Pauksztello, D. Ploog, Discrete derived categories I: homomorphisms, autoequivalences and t-structures. - arXiv:1312.5203 (2013). 
[8] A. Zimmermann, Self-equivalences of the derived category of Brauer tree algebras with exceptional vertex. - An. Ştiinţ. Univ. Ovidius Constanţa Ser. Mat. 9(1), 139-148 (2001).

[9] M. Schaps, E. Zakay-Illouz, Braid group action on the refolded tilting complex of the Brauer star algebra. — Proc. ICRA IX, Beijing 2, 434-449 (2002).

[10] P. Seidel, R. Thomas, Braid group actions on derived categories of coherent sheaves. Duke Math. J. 108(1), 37-108 (2001).

[11] M. Khovanov, P. Seidel. Quivers, Floer cohomology, and braid group actions. - J. Amer. Math. Soc. 15(1), 203-271 (2002).

[12] I. Muchtadi-Alamsyah, Braid action on derived category of Nakayama algebras. Comm. Algebra 36(7), 2544-2569 (2008).

[13] A. Zvonareva, On the derived Picard group of the Brauer star algebra. arXiv:1401.6952 (2014).

[14] Y. Volkov, A. Zvonareva, On standard derived equivalences of orbit categories. arXiv:1501.00709 (2015).

[15] A. Efimov, Braid group actions on triangulated categories. - The Möbius contest paper (2007).

[16] J. Rickard, Derived equivanences as derived functors. — J. London Math. Soc. 43(1), 37-48 (1991).

[17] B. Keller, Deriving DG categories. - Ann. Sci. École Norm. Sup. (4) 27(1), 63-102 (1994).

[18] J. Rickard, Morita theory for derived categories. — J. London Math. Soc. 39(3), 436-456 (1989).

[19] M. Bolla Isomorphisms between endomorphism rings of progenerators. - J. Algebra 87(1), 261-281 (1984).

[20] M. Linckelmann, Stable equivalences of Morita type for self-injective algebras and pgroups. - Math. Z. 223(1), 87-100 (1996).

[21] R. D. Pollack, Algebras and their automorphism groups. - Comm. Algebra 17(8), 18431866 (1989).

[22] F. Guil-Asensio, M. Saorín. The group of outer automorphisms and the Picard group of an algebra. - Algebr. Represent. Theory 2(4), 313-330 (1999).

[23] K. Erdmann, T. Holm, Twisted bimodules and Hochschild cohomology for self-injective algebras of class $A_{n}$. - Forum Math. 11 (2), 177-201 (1999).

[24] E. Brieskorn, K. Saito, Artin-gruppen und Coxeter-gruppen. - Invent. Math. 17(4), 245-271 (1972). 
[25] R. Charney, D. Peifer, The $K(\pi, 1)$-conjecture for the affine braid group. - Comment. Math. Helv. 78(3), 584-600 (2003).

[26] D. Allcock, Braid pictures for Artin groups. — Trans. Amer. Math. Soc. 354(9), 34553474 (2002).

[27] J. S. Birman, Mapping class groups and their relationship to braid groups. - Comm. Pure Appl. Math. 22(2), 213-238 (1969).

[28] R. C. Lyndon, P. E. Schupp, Combinatorial group theory. - Ergebnisse der Math., Springer, Berlin 89 (1977). 